\title{
Stable ergodicity and accessibility for certain partially hyperbolic diffeomorphisms with bidimensional center leaves
}

\author{
Vanderlei Horita* and Martin Sambarino*
}

\begin{abstract}
We consider classes of partially hyperbolic diffeomorphism $f: M \rightarrow M$ with splitting $T M=E^{s} \oplus E^{c} \oplus E^{u}$ and $\operatorname{dim} E^{c}=2$. These classes include for instance (perturbations of) the product of Anosov and conservative surface diffeomorphisms, skew products of surface diffeomorphisms over Anosov, partially hyperbolic symplectomorphisms on manifolds of dimension four with bidimensional center foliation whose center leaves are all compact. We prove that accessibility holds in these classes for $C^{1}$ open and $C^{r}$ dense subsets and moreover they are stably ergodic.
\end{abstract}

Mathematics Subject Classification (2010). 37D30; 37A25, 37C40.

Keywords. Accessibility, ergodicity, stable ergodicity, partial hyperbolicity.

\section{Introduction}

Ergodicity plays a fundamental role in Dynamics (and in Probability and Physics) since L. Boltzmann stated the "ergodic hypothesis" which says (roughly speaking) that in an evolution law time average and space average are equal. More precisely, we say that a dynamical system $f: M \rightarrow M$ preserving a finite measure $m$ is ergodic (with respect to $m$ ) if any invariant set has zero measure or its complement has zero measure.

E. Hopf [19] proved the ergodicity of the geodesic flow on surfaces of negative curvature. This was extended by Anosov to the geodesic flow on compact manifolds with negative curvature in a cornerstone paper in dynamics [3]. He also proved that conservative (today called) Anosov $C^{1+\alpha}$ diffeomorphisms are ergodic. And, since Anosov diffeomorphisms are open, the above implies that conservative Anosov systems are stably ergodic. We say that a $C^{r}$ diffeomorphism $f: M \rightarrow M$ preserving a measure $m$ is $C^{r}$ stably ergodic if any sufficiently small $C^{r}$ perturbation of $f$ preserving $m$ is ergodic.

${ }^{*}$ Work partially supported by CAPES, FAPESP, PRONEX and PROSUL, Brazil; Palis-Balzan project; CSIC-Dynamic Group 618-Uruguay and MathAmSud project PhySeCo. 
In a seminal work, Grayson, Pugh, and Shub [18] proved that the time one map of the geodesic flow of a hyperbolic surface is $C^{2}$ stably ergodic. Afterwards, Ch. Pugh and M. Shub recovered (in some sense) Smale's program in the sixties about stability and genericity by restricting to partially hyperbolic diffeomorphisms on manifolds preserving the Lebesgue measure and replacing structural stability by stable ergodicity. They conjectured that among $C^{2}$ partially hyperbolic diffeomorphism preserving the Lebesgue measure $m$, stable ergodicity holds in an open and dense set. They proved important results in this direction and they proposed a program as well (see [25,26], and [27]). The main conjecture is:

Conjecture 1.1 ([27]). On any compact manifold, ergodicity holds for an open and dense set of $C^{2}$ volume preserving partially hyperbolic diffeomorphisms.

This conjecture splits into two conjectures where accessibility (see Definition 1.6) plays a key role:

Conjecture 1.2 ([27]). Accessibility holds for an open and dense set of $C^{2}$ partially hyperbolic diffeomorphism, volume preserving or not.

Conjecture 1.3 ([27]). A partially hyperbolic $C^{2}$ volume preserving diffeomorphism with the essential accessibility property is ergodic.

They also proved [27] a result in the direction of the third conjecture: A partially hyperbolic $C^{2}$ volume preserving diffeomorphism, dynamically coherent, center bunched, and with the essential accessibility property is ergodic. Since then, a lot of research on the field has been done. See the surveys [7,31,37], and [12] for an account on this progress during the last decades.

In [10], K. Burns and A. Wilkinson improved a lot Pugh-Shub result in two directions: dynamically coherence is not needed and the center bunching condition is much milder than originally stated.

The key fact thus to obtain ergodicity is accessibility. In [14] it is proved that accessibility holds for a $C^{1}$ open and dense subset of $C^{r}$ partially hyperbolic diffeomorphism, volume preserving or not. When the center bundle has dimension one, it is proved in [32] that accessibility holds for a $C^{1}$ open and $C^{r}$ dense subset of $C^{r}$ partially hyperbolic volume preserving diffeomorphism (later extended to the non-volume preserving case in [5]). This in particular implies the main conjecture in its full generality when the center dimension is one.

There has been in the last years a great advance to the main conjecture in the $C^{1}$ topology. In fact in [30] it is proved that stably ergodicity is $C^{1}$ dense when the center dimension is two. And recently, an outstanding result has been obtained by A. Avila, S. Crovisier, and A. Wilkinson [1]: stable ergodicity is $C^{1}$ dense in any case (without any assumption on the dimension of the center bundle).

These results depends heavily on perturbation techniques available in the $C^{1}$ topology and not known on higher topologies. The $C^{r}$ denseness of stable ergodicity, $r \geq 2$, is a complete different problem. Little is known in this case 
when the center bundle has dimension greater than one. In [8], the authors prove $C^{r}$ density of stable ergodicity for group extensions over Anosov diffeomorphisms. A remarkable result has been obtained by F. Rodriguez Hertz [29] for certain automorphisms of the torus $\mathbb{T}^{d}$. Also, in [35] are given two examples that can be $C^{r}, r \geq 2$, approximated by stable ergodic ones. And very recently Z. Zhang [41] obtained $C^{r}$ density of stable ergodicity for volume preserving diffeomorphisms satisfying some pinching condition and a certain type of dominated splitting on the center. A. Avila and M. Viana have announced $C^{1}$ openness and $C^{r}$ density for certain skew product of surfaces diffeomorphisms over Anosov and our work might have some overlap with theirs although our methods are different.

Our aim in this paper is to contribute to the $C^{r}$ denseness of stable ergodicity, in particular when the center dimension is two. We prove that for large classes of $C^{r}$ partially hyperbolic volume preserving diffeomorphisms with two dimensional center bundle, stable ergodicity holds in $C^{r}$ dense subsets. Precise statements are given in Section 1.2. However, just to give a flavor of them let us state a particular case (see Theorems 4A and 4B).

Theorem 1. Ergodicity holds in $C^{1}$ open and $C^{r}$ dense subset in the following settings:

- Skew products of conservative surfaces diffeomorphisms over conservative Anosov diffeomorphisms.

- Partially hyperbolic symplectomorphisms on $(M, \omega)$ where $\operatorname{dim} M=4$ having a bidimensional center foliation whose leaves are all compact.

The main tool we use to prove the ergodicity is accessibility. Thus, we have to prove that accessibility holds in a $C^{1}$ open and $C^{r}$ dense subset in the setting we are working with. The main idea is to use results on conservative surface dynamics to show that generically one gets accessibility. Indeed, when the center dimension is two and we look to the accessibility class inside a (periodic) compact center leaf we have three possibilities: it has zero, one or two topological dimensions. We prove that generically (see Theorem 2) zero dimensional accessibility classes do not exist. We will use to the full extent results on conservative surface dynamics to prove that also generically one-dimensional accessibility classes do not exist and therefore the accessibility classes are open on the center leaf and so there is just one accessibility class.

1.1. Setting. Let $f: M \rightarrow M$ be a diffeomorphism where $M$ is a compact riemannian manifold without boundary. We say that $f$ is partially hyperbolic if the tangent bundle splits into three subbundles $T M=E^{s} \oplus E^{c} \oplus E^{u}$ invariant under the tangent map $D f$ and such that:

- There exists $0<\lambda<1$ such that

$$
\left\|D f_{/ E^{s}}\right\|<\lambda \quad \text { and } \quad\left\|D f_{/ E^{u}}^{-1}\right\|<\lambda .
$$


- For every $x \in M$ we have

$$
\frac{\left\|D f_{/ E_{x}^{s}}\right\|}{m\left\{D f_{/ E_{x}^{c}}\right\}}<1 \quad \text { and } \quad \frac{\left\|D f_{/ E_{x}^{c}}\right\|}{m\left\{D f_{/ E_{x}^{u}}\right\}}<1
$$

where $m\{A\}$ is the co-norm of $A$, i.e., $m\{A\}=\left\|A^{-1}\right\|^{-1}$.

By continuity of $D f$ and the compactness of $M$, there is a positive constant $\eta<1$ such that the inequalities in the last item hold for $\eta$ instead of 1 . In other words, $E^{s}$ is uniformly contracted, $E^{u}$ is uniformly expanded and the behaviour of $E^{c}$ is between both.

It is well known that the subbundles $E^{s}$ and $E^{u}$ uniquely integrate to two foliations $\mathscr{F}^{s}=\mathscr{F}_{f}^{s}$ and $\mathscr{F}^{u}=\mathscr{F}_{f}^{u}$ called the stable and unstable foliation respectively. We denote by $\mathcal{F}^{\sigma}(x)(\sigma=s, u)$ the leaf of the foliation through the point $x$.

On the other hand it is not always true that the center subbundle $E^{c}$ is integrable. We say that the partially hyperbolic diffeomorphism $f$ is dynamically coherent if the bundles $E^{s} \oplus E^{c}$ and $E^{c} \oplus E^{u}$ integrate to invariant foliations $\mathcal{F}^{c s}$ and $\mathscr{F}^{c u}$ called the center stable and center unstable foliations respectively. In particular $E^{c}$ integrates to a (normally hyperbolic) invariant foliation $\mathscr{F}^{c}$. Moreover, $\mathscr{F}^{c}$ and $\mathscr{F}^{s}$ subfoliates $\mathscr{F}^{c s}$ and $\mathscr{F}^{c}$ and $\mathscr{F}^{u}$ subfoliates $\mathscr{F}^{c u}$, see [9].

We say the center foliation is $r$-normally hyperbolic $(r \geq 1)$ if the following holds:

$$
\frac{\left\|D f_{/ E_{x}^{s}}\right\|}{m\left\{D f_{/ E_{x}^{c}}\right\}^{r}}<1 \text { and } \frac{\left\|D f_{/ E_{x}^{c}}\right\|^{r}}{m\left\{D f_{/ E_{x}^{u}}^{u}\right\}}<1 .
$$

If $f$ is of class $C^{r}$ and the center foliation is $r$-normally hyperbolic then the leaves of $\mathscr{F}^{c}$ are of $C^{r}$ class (see [20]).

Partially hyperbolic diffeomorphisms are $C^{1}$ open. In order to assure that dynamically coherence also holds for $C^{1}$ systems nearby we have to require plaque expansiveness. This is technical and we will not define it here, we refer to [20] (however, if $\mathscr{F}^{c}$ is a $C^{1}$ foliation or all leaves of $\mathscr{F}^{c}$ are compact then the center foliation is plaque expansive). The results on [20] (see Theorem 7.4) assure that a normally hyperbolic and plaque expansive foliation $\mathcal{F}_{f}^{c}$ of a diffeomorphism $f$ is structurally stable, that is, there exist a neighborhood of $U(f)$ and a homeomorphism $h: M \rightarrow M$ such that for $g \in \mathcal{U}(f)$ there exists a (normally hyperbolic) foliation $\mathcal{F}_{g}^{c}$ such that $h\left(\mathscr{F}_{f}^{c}(x)\right)=\mathscr{F}_{g}^{c}(h(x))$ and $h\left(\mathscr{F}_{f}^{c}(f(x))\right)=\mathscr{F}_{g}^{c}(g(h(x)))$. This result implies that partially hyperbolic diffeomorphisms, dynamically coherent with center foliation plaque expansive are $C^{1}$ open.

We also say that a partially hyperbolic diffeomorphism $f$ is center bunched if:

$$
\left\|D f_{/ E_{x}^{s}}\right\| \frac{\left\|D f_{/ E_{x}^{c}}\right\|}{m\left\{D f_{/ E_{x}^{c}}\right\}}<1 \quad \text { and } \quad \frac{\left\|D f_{/ E_{x}^{c}}\right\|}{m\left\{D f_{/ E_{x}^{c}}\right\}} \frac{1}{m\left\{D f_{/ E_{x}^{u}}\right\}}<1 .
$$

This bunching condition is as in [10] where they improve substantially the one stated by Pugh-Shub originally. Notice that the bunching condition is also $C^{1}$ open. 
We say that a partially hyperbolic diffeomorphism $f: M \rightarrow M$ dynamically coherent has Global Product Structure (GPS for short) if there is a covering $\pi$ : $\widetilde{M} \rightarrow M$ and a lift $\tilde{f}: \widetilde{M} \rightarrow \widetilde{M}$ of $f$ such that when we lift the invariant foliations (stable, unstable, center-stable, and center-unstable) to $\widetilde{M}$ we have for any $\widetilde{x}, \tilde{y}$ in $\widetilde{M}$ :

$$
\#\left\{\widetilde{F}^{c s}(\widetilde{x}) \cap \widetilde{F}^{u}(\tilde{y})\right\}=1 \quad \text { and } \quad \#\left\{\widetilde{F}^{c u}(\widetilde{x}) \cap \widetilde{F}^{s}(\tilde{y})\right\}=1 .
$$

The GPS also implies that, in the covering $\widetilde{M}$ every stable leaf intersects in one point every center leaf inside a center stable leaf, i.e. if $\tilde{y}, \tilde{z} \in \widetilde{F}^{c s}(\tilde{x})$ then $\#\left\{\widetilde{F}^{s}(\tilde{y}) \cap \widetilde{F}^{c}(\tilde{z})\right\}=1$. The same in center-unstable leaves in the covering. In particular, if $y, z \in \mathscr{F}^{c s}(x)$, then $\mathscr{F}^{s}(y) \cap \mathscr{F}^{c}(z) \neq \emptyset$, although this intersection might not be unique.

One of the main property of the GPS is that it allow us to define a global projection (in $\widetilde{M}$ ) onto a given center stable manifold along the holonomy of the unstable foliation. We denote by $\mathscr{E P} \mathcal{S}$ the $C^{1}$ interior of the set of partially hyperbolic diffeomorphisms dynamically coherent having GPS.

Definition 1.4. Let $M$ be a compact riemannian manifold without boundary and let $r \geq 1$. We denote by $\mathcal{E}^{r}=\mathcal{E}^{r}(M)$ the set of $C^{r}$ diffeomorphisms $f: M \rightarrow M$ (with the $C^{r}$ topology) such that

- $f$ is partially hyperbolic;

- $f$ is dynamically coherent;

- the center foliation is $r$-normally hyperbolic and plaque expansive;

- $f$ is center bunched;

- $f \in \mathcal{E P} \&$; and

- the set of center leaves that are compact and $f$-periodic are dense in $M$.

We remark that $\varepsilon^{r}$ is $C^{1}$ open (and hence $C^{r}$ open as well).

Examples. Here we give some examples of diffeomorphism in $\mathcal{E}^{r}$. We restrict ourselves where the center dimension is two.

(1) Perturbation of product of diffeomorphisms: Let $g: S \rightarrow S$ be a $C^{r}$ diffeomorphism of a compact surface and let $f: N \rightarrow N$ be a transitive Anosov diffeomorphism. If the contraction and expansion of $f$ are strong enough we get that $f \times g \in \mathcal{E}^{r}(M)$ where $M=N \times S$. Notice that the center foliation consists of compact manifolds homeomorphic to $S$. In particular, the space of center leaves is homeomorphic to $N$ and the dynamics of the center leaves is conjugated to the Anosov diffeomorphism $f: N \rightarrow N$ and hence (lifting to the covering $\widetilde{M}=\widetilde{N} \times S$ where $\widetilde{N}$ is the universal covering of $N$ ) $f \times g$ has GPS (and indeed belongs to $\mathscr{P} \mathcal{P}$ ). In case $g=$ id then automatically $f \times g \in \mathcal{E}^{r}$ for any $r \geq 1$. 
(2) Skew products over Anosov: Let $f: N \rightarrow N$ be a $C^{r}$ (transitive) Anosov diffeomorphism and consider $S$ a compact surface. Let $U \subset \operatorname{Diff}^{r}(S)$ be the open set such that if $h \in \mathcal{U}$ then $f \times h$ is partially hyperbolic with center fiber $\{x\} \times S$, center bunched, and $r$-normally hyperbolic. Let $g: N \rightarrow \mathcal{U}$ be a continuous map. For $x \in N$ let's denote by $g_{x}$ the diffeomorphism $g(x): S \rightarrow S$. For such a map $g$ consider the skew product $F=f \times \times_{s p} g: N \times S \rightarrow N \times S$ by

$$
F(x, y)=\left(f(x), g_{x}(y)\right) \text {. }
$$

Notice that the center foliation is $\{\{x\} \times S, x \in N\}$ and the dynamics of the center leaves is the one of the Anosov $f: N \rightarrow N$. It follows that $F$ has GPS (and any $C^{r}$ perturbation has GPS as well) and the periodic center leaves are dense. Thus, we have that $F \in \mathcal{E}^{r}(N \times S)$. We may consider thus perturbations of $F$ in $\mathcal{E}^{r}$ and also perturbations in the skew product setting. For this, let $\mathscr{E}=\{g: N \rightarrow \mathcal{U}$ : continuous $\}$, where $g, \tilde{g} \in \mathcal{E}$ are close if $g_{x}, \widetilde{g_{x}}$ are $C^{r}$ close for all $x \in N$. We denote by $\mathcal{E}_{s p}^{r}$ the set of skew products $f \times_{s p} g$ with $g \in \mathscr{G}$.

If $\omega$ is an area (symplectic) form on $S$ we denote by $\mathcal{E}_{s p, \omega}^{r}$ the set of skew products as above where $g_{x}$ preserves $\omega$ for all $x \in N$. Notice that in general $F \in \mathcal{E}_{s p, \omega}^{r}$ does not preserves volume. If the base map $f: N \rightarrow N$ preserves a volume form $\eta$ then $F \in \mathcal{E}_{s p, \omega}^{r}$ preserves the volume form in $N \times S$ given by $\eta \times \omega$.

(3) Let $f: M \rightarrow M$ be partially hyperbolic diffeomorphism of a four dimensional manifold, dynamically coherent whose center leaves are all compact and bidimensional. Results of Gogolev [17] (see also Carrasco [11] and Bohnet [6]) implies that center foliation is uniformly compact (that is, the leaves have finite holonomy), and that fibers over an Anosov diffeomorphism on the torus $\mathbb{T}^{2}$. In particular it has GPS and the periodic center leaves are dense.

(4) (Perturbation of) the product of the time $t$ of an Anosov suspension and a rotation: Consider $f: N \rightarrow N$ the time $t$ map of the suspension of a transitive Anosov diffeomorphism and let $R: \mathbb{S}^{1} \rightarrow \mathbb{S}^{1}$ be a rotation. Let $f \times R: N \times \mathbb{S}^{1} \rightarrow$ $N \times \mathbb{S}^{1}$. It is not difficult to see that belongs to $\mathcal{E}^{r}$ for any $r$ as long as $f$ is $C^{r}$.

(5) (Perturbation of) the product of time maps of Anosov suspensions: Let $f, g$ be time maps of the suspensions of a transitive Anosov diffeomorphisms. Then $f \times g$ belongs to $\varepsilon^{r}$.

Remark 1.5. We considered time maps of Anosov suspensions so that there is a lift with GPS. There are time-1 map of Anosov flows without GPS, for instance, time-1 map of the geodesic flow in a surface of negative curvature.

1.2. Statements of Results. We denote by $\mathcal{E}_{m}^{r}(M)$ the set of diffeomorphisms in $\mathcal{E}^{r}$ preserving a volume form $m$ on $M$, and by $\mathcal{E}_{\omega}^{r}$ the ones in $\mathcal{E}^{r}$ preserving a symplectic form $\omega$ on $M$. And recall that $\mathcal{E}_{s p}^{r}(M), \mathcal{E}_{s p, \omega}^{r}(M)$ are the skew products over Anosov diffeomorphism on $M=N \times S$ where $S$ is a compact surface and $\omega$ is an area form on $S$. 
Our results mainly concerns accessibility, so let us introduce the concept.

Definition 1.6. Let $f: M \rightarrow M$ be a partially hyperbolic diffeomorphism in $\mathcal{E}^{r}$. A su-path is a continuous curve $\alpha:[0,1] \rightarrow M$ such that there exists a partition $0=t_{0}<t_{1}<\cdots<t_{n}=1$ such that $\alpha\left(\left[t_{i}, t_{i+1}\right]\right)$ is contained either in a leaf of $\mathcal{F}^{s}$ or in a leaf of $\mathscr{F}^{u}$. The relation $x \sim y$ if there exists a $s u$-path from $x$ to $y$ is an equivalence relation on $M$.

For a point $x \in M$ the accessibility class $A C(x)$ of $x$ is:

$$
A C(x)=\{y \in M: \text { there is a su-path from } x \text { to } y\} .
$$

We say that $f$ is accessible if $A C(x)=M$ for some $x$ (and hence for all $x \in M$ ). On the other hand, we say the accessibility class $A C(x)$ is trivial if $A C(x) \cap \mathscr{F}^{c}(x)$ is totally disconnected.

Our first result concerns trivial accessibility classes (with no restriction on the dimension of the center leaves):

Theorem 2. Let $r \geq 2$ and let $\mathcal{E}$ denote $\mathcal{E}^{r}, \mathcal{E}_{m}^{r}, \mathcal{E}_{\omega}^{r}, \mathcal{E}_{s p}^{r}$ or $\mathcal{E}_{s p, \omega}^{r}$. Then, the set $\mathcal{R}_{0}$ of diffeomorphisms in $\mathcal{E}$ having no trivial accessibility classes is $C^{1}$ open and $C^{r}$ dense.

The next result gives a condition to assure accessibility when the center leaves have dimension two. Recall that a sink is an attracting periodic orbit. Call a diffeomorphism $g: S \rightarrow S$ sinkless if it has no sinks. Define a subset $\mathcal{E}_{A}^{r}$ of $\mathcal{E}^{r}$ by $f \in \mathcal{E}_{A}^{r}$ if there is a periodic center leaf $L=f^{k}(L)$ for which $f^{k}$ restricted to $L$ is both Axiom $A$ and sinkless. Note that $\mathcal{E}_{A}^{r}$ is a $C^{1}$ open subset of $\mathcal{E}^{r}$.

Theorem 3A. Assume that $r \geq 2$ and $\operatorname{dim} E^{c}=2$. Then, there exists $\widehat{\mathcal{R}} \subset \mathcal{E}_{A}^{r}$ which is $C^{1}$ open and $C^{r}$ dense in $\mathcal{E}_{A}^{r}$ such that any $f \in \widehat{\mathcal{R}}$ is accessible.

In the conservative setting define $\mathcal{E}_{A, m}^{r}:=\mathcal{E}_{A}^{r} \cap \mathcal{E}_{m}^{r}$ and note that this is a $C^{1}$ open subset of $\mathcal{E}_{m}^{r}$.

Theorem 3B. Assume that $r \geq 2$ and $\operatorname{dim} E^{c}=2$. Then, there exists $\widehat{\mathcal{R}} \subset \mathcal{E}_{A, m}^{r}$ which is $C^{1}$ open and $C^{r}$ dense in $\mathcal{E}_{A, m}^{r}$ such that any $f \in \widehat{\mathcal{R}}$ is accessible. In particular any $f \in \hat{\mathcal{R}}$ is stably ergodic.

Above, we considered a sinkless diffeomorphism $f_{\mid L}^{k}$. By replacing $f$ with its inverse, analogous results also hold when $f_{\mid L}^{k}$ is both Axiom A and "sourceless".

Finally, the next two results says that accessibility holds generically in the case of skew products or when a symplectic form is preserved (they imply Theorem 1):

Theorem 4A. Assume that $r \geq 2$ and $\operatorname{dim} E^{c}=2$ and consider the space $\mathcal{E}_{s p, \omega}^{r}$ of skew-products preserving an area form $\omega$. Then, there exists $\mathcal{R} \subset \mathcal{E}_{s p, \omega}^{r}$ which is $C^{1}$ open and $C^{r}$ dense such that all diffeomorphism in $\mathcal{R}$ are accessible. In particular, if the base map preserve a volume form $\eta$, any $f \in \mathcal{R}$ is stably ergodic with respect to the volume induced by $\eta \times \omega$. 
Theorem 4B. Assume that $r \geq 2$ and $\operatorname{dim} E^{c}=2$ and consider the subspace $\mathcal{E}_{\omega}^{r}$ of $\mathcal{E}^{r}$ formed by those preserving a symplectic form $\omega$. Then, there exists $\mathcal{R} \subset \mathcal{E}_{\omega}^{r}$ which is $C^{1}$ open and $C^{r}$ dense in $\mathcal{E}_{\omega}^{r}$ such that all diffeomorphism in $\mathcal{R}$ are accessible. In particular they are stable ergodic with respect to the volume induced by the symplectic form $\omega$.

Organization of the paper. In Section 2 we give general facts concerning accessibility classes and some results regarding its structure when the center has dimension two. In Section 3 we prove some perturbation results in order to obtain later some generic results on the accessibility classes. Section 4 is devoted to prove Theorem 2. The accessibility classes of periodic points are studied in Section 5, where it is proved that generically, when the center subbundle has dimension two, the accessibility classes of hyperbolic periodic points or elliptic (when we restrict to an invariant center leaf) are open. Theorem 3A and Theorem 3B are proved in Section 6. Finally, in Section 7 we prove Theorem 4A and Theorem 4B.

Acknowledgements. We wish to thank C. Bonatti, P. Le Calvez, A. Koropecki, E. Pujals, and M. Viana for useful conversations and especially R. Potrie for reading a draft version of the paper and giving some insightful comments. We also are very grateful to the referee whose comments and corrections helped us to improve the paper.

\section{Basic facts on accessibility}

In this section we establish some basic results on accessibility. We assume that $f: M \rightarrow M$ belongs to $\mathcal{E}^{r}, r \geq 2$, although some remarks hold in general.

When $y \in \mathscr{F}^{s}(x)$ denote by $\Pi_{f}^{s}\left(\mathscr{F}^{c}(x), \mathscr{F}^{c}(y)\right)$ the (local) holonomy map from a neighborhood of $x$ in $\mathscr{F}^{c}(x)$ to a neighborhood of $y$ in $\mathscr{F}^{c}(y)$ along the stable leaves (inside $\left.\mathscr{F}^{c s}(x)=\mathscr{F}^{c s}(y)\right)$ ). This map is well defined since the leaves of $\mathscr{F}^{s}$ are simple connected. By [28] this holonomy map is of class $C^{1}$, i.e. the holonomy map inside center stable leaves along stable leaves is $C^{1}$. The same holds for center unstable leaves and holonomy along unstable leaves and so, for $\Pi_{f}^{u}\left(\mathcal{F}^{c}(x), \mathcal{F}^{c}(y)\right)$.

Recall that a diffeomorphism $f$ in $\mathcal{E}^{r}$ has Global Product Structure, that is, there exists a covering map $\pi: \widetilde{M} \rightarrow M$ such that denoting by $\widetilde{F}^{*}, *=s, u, c s, c u, c$ the lift of the stable, unstable, center stable, center unstable, and center leaves respectively, then for every $\widetilde{x}, \tilde{y} \in \widetilde{M}$ we have:

$$
\#\left\{\widetilde{F}^{c s}(\widetilde{x}) \cap \widetilde{F}^{u}(\widetilde{y})\right\}=1 \quad \text { and } \quad \#\left\{\widetilde{F}^{c u}(\widetilde{x}) \cap \widetilde{F}^{s}(\widetilde{y})\right\}=1 .
$$

This allows to define a continuous map

$$
\Pi_{\widetilde{x}}^{u}: \widetilde{M} \rightarrow \widetilde{\mathscr{F}} c s(\widetilde{x}),
$$


defined by the holonomy map along unstable leaves:

$$
\Pi_{\widetilde{x}}^{u}(\widetilde{z})=\widetilde{\mathscr{F}}^{u}(\widetilde{z}) \cap \widetilde{\mathscr{F}}^{c s}(\widetilde{x})
$$

If we restrict the map $\Pi_{\widetilde{x}}^{u}$ to $\widetilde{\mathscr{F}}^{c u}(\tilde{x})$, we have that $\Pi_{\widetilde{x}}^{u}\left(\widetilde{\mathcal{F}}^{c u}(\tilde{x})\right)=\widetilde{\mathscr{F}}^{c}(\tilde{x})$. In an analogous way we define $\Pi_{\tilde{x}}^{s}$ the holonomy along stable leaves. And also we define $\Pi_{\widetilde{x}}^{s u}: \widetilde{M} \rightarrow \widetilde{\mathscr{F}}^{c}(\tilde{x})$ by

$$
\Pi_{\widetilde{x}}^{s u}=\Pi_{\widetilde{x}}^{s} \circ \Pi_{\tilde{x}}^{u}
$$

Note that, in general, $\Pi_{\widetilde{x}}^{u s} \neq \Pi_{\widetilde{x}}^{s u}$.

Recall that we have defined the accessibility class of $x \in M$ as

$$
A C(x)=\{y \in M: \text { there is a su-path from } x \text { to } y\} .
$$

We define the center accessibility class of $x$ as $C(x)=A C(x) \cap \mathcal{F}^{c}(x)$.

The same definitions for $\widetilde{M}:$ for $\tilde{x} \in \widetilde{M}$ its accessibility class is

$$
\widetilde{A C}(\tilde{x})=\{\tilde{y} \in \widetilde{M}: \text { there is a } s u \text {-path from } \tilde{x} \text { to } \tilde{y}\}
$$

and $\widetilde{C}(\tilde{x})=\widetilde{A C}(\widetilde{x}) \cap \widetilde{\mathcal{F}}^{c}(\tilde{x})$.

Let us observe that if

$$
\tilde{x}, \widetilde{z} \in \widetilde{M} \text { with } \widetilde{z} \in \widetilde{A C}(\widetilde{x}) \Longrightarrow \Pi_{\widetilde{x}}^{s u}(\widetilde{z}) \in \widetilde{C}(\tilde{x})
$$

Lemma 2.1. Let $\tilde{x} \in \widetilde{M}$ and set $x=\pi(\tilde{x})$. Then

- $\pi(\widetilde{A C}(\tilde{x}))=A C(x)$.

- $\pi(\widetilde{C}(\tilde{x})) \subset C(x)$.

Proof. Note that the projection of a $s u$-path in $\widetilde{M}$ is a $s u$-path in $M$. Then, $\pi(\widetilde{A C}(\tilde{x})) \subset A C(x)$. Reciprocally, the lift of a $s u$-path in $M$ is a $s u$-path in $\widetilde{M}$. The second part also follows easily:

$$
\begin{aligned}
\pi(\widetilde{C}(\tilde{x})) & =\pi\left(\widetilde{A C}(\widetilde{x}) \cap \widetilde{\mathscr{F}}^{c}(\tilde{x})\right) \subset \pi(\widetilde{A C}(\tilde{x})) \cap \pi\left(\widetilde{\mathscr{F}}^{c}(\tilde{x})\right) \\
& =A C(x) \cap \mathcal{F}^{c}(x)=C(x) .
\end{aligned}
$$

The proof is complete.

Lemma 2.2. The following are equivalent:
(a) $A C(x)$ is an open subset.
(b) $A C(x)$ has non-empty interior.
(c) $C(x)$ is an open subset of $\mathscr{F}^{c}(x)$
(d) $C(x)$ has non-empty interior (in $\left.\mathcal{F}^{c}(x)\right)$. 
Proof. Notice that, by continuity of $\mathcal{F}^{s}$, if $U$ is an open set in $M$ then the saturation by stable leaves, i.e. $\cup_{x \in U} \mathcal{F}^{s}(x)$ is also open in $M$ and the same for the saturation by unstable leaves. Moreover, from the local product structure due the partially hyperbolic structure, given an open set $V$ in a center leaf, its saturation by stable and unstable leaf is also an open set in $M$. From these simple facts the lemma follows.

The same is true for the lift and also equivalent to the above:

Lemma 2.3. The following are equivalent:

(a) $\widetilde{A C}(\widetilde{x})$ is an open subset.

(b) $\widetilde{A C}(\widetilde{x})$ has non-empty interior.

(c) $\widetilde{C}(\widetilde{x})$ is an open subset of $\widetilde{F}^{c}(x)$.

(d) $\widetilde{C}(\widetilde{x})$ has non-empty interior (in $\widetilde{F}^{c}(x)$ ).

(e) $C(x)$ is an open subset of $\mathscr{F}^{c}(x)$.

Proof. The proof of the equivalence among the first four items is analogous as the previous lemma. Let's see the equivalence with the last one. If $\widetilde{A C}(\widetilde{x})$ is open we get, by Lemma 2.1 and since $\pi$ is a covering map, that $A C(x)=\pi(\widetilde{A C}(\widetilde{x}))$ is open and hence $C(x)$ is open by lemma above. On the other hand, if $C(x)$ is open in $\mathscr{F}^{c}(x)$ then $A C(x)$ is also open in $M$. It follows that $\pi^{-1}(A C(x))$ is open in $\widetilde{M}$. Since $\pi^{-1}(A C(x))=\cup_{\beta} \beta(\widetilde{A C}(\widetilde{x}))$ where $\beta$ runs over all covering transformations. It follows, for some $\beta$ covering transformation, that $\beta(\widetilde{A C}(\tilde{x}))$ has non-empty interior and so $\widetilde{A C}(\widetilde{x})$ has non-empty interior.

Lemma 2.4. For any $z \in M$ and any center leaf $\mathscr{F}^{c}(x)$ we have that $A C(z) \cap$ $\mathscr{F}^{c}(x) \neq \emptyset$. In particular, $f$ is accessible if and only if for some $x$ it holds that $C(x)=\mathscr{F}^{c}(x)$.

Proof. The Global Product Structure implies in particular that for any $z$ and $x$ we have that:

$$
\mathscr{F}^{u}(z) \cap \mathscr{F}^{c s}(x) \neq \emptyset \quad \text { and } \quad \mathscr{F}^{c s}(x)=\bigcup_{y \in \mathscr{F}^{c}(x)} \mathscr{F}^{s}(y)
$$

which yields $A C(z) \cap \mathscr{F}^{c}(x) \neq \emptyset$. The second part follows immediately.

Recall that for $y \in \mathscr{F}^{s}(x)$ the (local) holonomy map $\Pi^{s}\left(\mathscr{F}^{c}(x), \mathscr{F}^{c}(y)\right)$ from a neighborhood $U_{x}^{c}$ of $x$ in $\mathscr{F}^{c}(x)$ to a neighborhood $U_{y}^{c}$ of $y$ in $\mathscr{F}^{c}(y)$ is a $C^{1}$ diffeomorphism. If $\gamma:[0,1] \rightarrow \mathscr{F}^{s}(x)$ is a path joining $x$ and $y$ then there exists a continuous map $\Gamma^{s}: U_{x}^{c} \times[0,1] \rightarrow \mathscr{F}^{c s}(x)$ such that:

- $\Gamma^{s}(z, t) \in \mathscr{F}^{s}(z) \forall t \in[0,1]$. In particular $\Gamma^{s}(z, t) \in A C(z)$.

- $\Gamma^{s}(z, 0)=z$ for all $z \in U_{x}^{c}$. 
- $\Gamma^{s}(z, 1)=\Pi^{s}\left(\mathcal{F}^{c}(x), \mathcal{F}^{c}(y)\right)(z) \in U_{y}^{c}$.

- $\Gamma_{0}^{s}: U_{x}^{c} \rightarrow U_{y}^{c}$ defined by $\Gamma_{0}^{s}(z)=\Gamma(z, 1)=\Pi^{s}\left(\mathscr{F}^{c}(x), \mathscr{F}^{c}(y)\right)(z)$ is a $C^{1}$ diffeomorphism.

The same holds when $y \in \mathscr{F}^{u}(x)$ considering $\Pi^{u}\left(\mathscr{F}^{c}(x), \mathscr{F}^{c}(y)\right)$ and also for the lift $\widetilde{f}: \widetilde{M} \rightarrow \widetilde{M}$. In particular, if $y \in A C(x)$ and $\gamma$ is $s u$-path joining $x$ to $y$, by finite composition of maps as above we get a map (see Figure 1)

$$
\Gamma: U_{x}^{c} \times[0,1] \rightarrow M
$$

such that

- $\Gamma(z, t) \in A C(z)$ for all $t \in[0,1]$.

- $\Gamma(z, 0)=z$ for all $z \in U_{x}^{c}$.

- $\Gamma(z, 1) \in U_{y}^{c}$.

- $\Gamma_{0}: U_{x}^{c} \rightarrow U_{y}^{c}$ defined by $\Gamma_{0}(z)=\Gamma(z, 1)$ is a $C^{1}$ diffeomorphism.

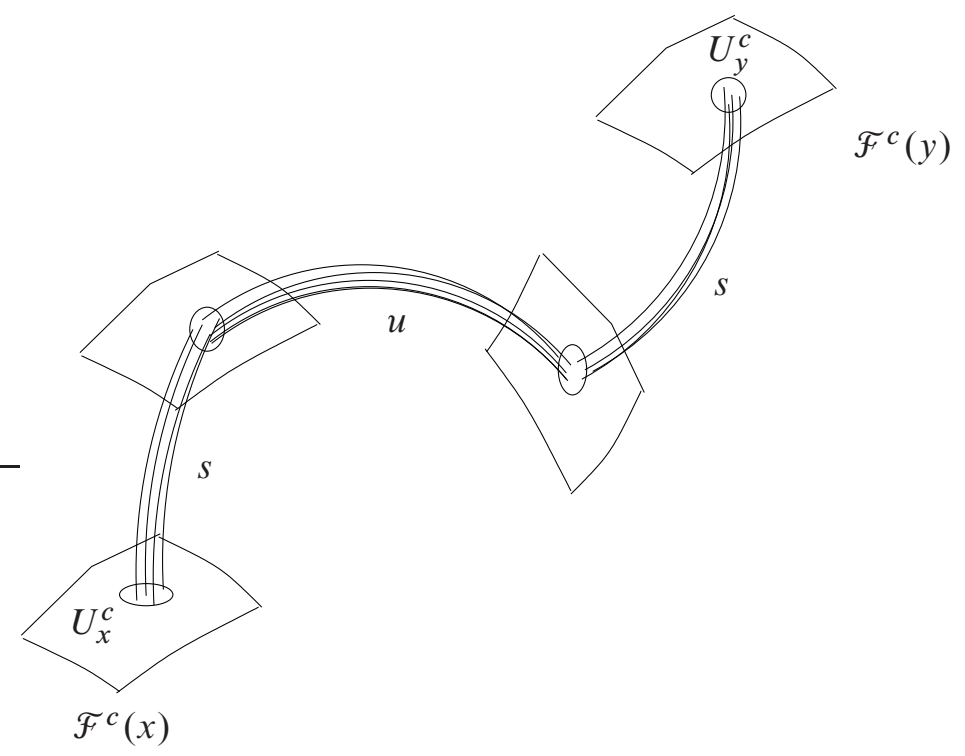

Figure 1. The map $\Gamma$.

In the same way, if $\tilde{y} \in \widetilde{A C}(\tilde{x})$ we have a map

$$
\widetilde{\Gamma}: U_{\widetilde{x}}^{c} \times[0,1] \rightarrow \widetilde{M}
$$

with the properties above. The above has important consequences. First, we recall the definition of a homogeneous subset. 
Definition 2.5. Let $X$ be a riemannian manifold. A subset $Z \subset X$ is said $C^{r}$-homogeneous if for every pair of points $x, y \in Z$ there are neighborhoods $U_{x}, U_{y} \subset X$ of $x$ and $y$, respectively, and a $C^{r}$-diffeomorphism $\phi: U_{x} \rightarrow U_{y}$ such that $\phi\left(U_{x} \cap Z\right)=U_{y} \cap Z$ and $\phi(x)=y$.

Thus, it follows straightforward from the definitions of $\Gamma$ and $\widetilde{\Gamma}$ that:

Lemma 2.6. $C(x)$ and $\widetilde{C}(\widetilde{x})$ are $C^{1}$-homogeneous.

Moreover, we have the following:

Lemma 2.7. Let $\tilde{x} \in \widetilde{M}$ and $\tilde{y} \in \widetilde{C}(\widetilde{x})$. Then there are neighborhoods $U_{\widetilde{x}}^{c}$ and $U_{\widetilde{y}}^{c}$ in $\widetilde{F}^{c}(x)$ and a continuous map $\widetilde{\Gamma}: U_{\widetilde{x}}^{c} \times[0,1] \rightarrow \widetilde{F}^{c}(\widetilde{x})$ such that

(1) $\tilde{\gamma}(\widetilde{z}, 0)=\tilde{z}$ for all $\tilde{z} \in U_{\widetilde{x}}^{c}$;

(2) $\tilde{\gamma}(\widetilde{z}, 1) \in U_{\widetilde{y}}^{c}$ for all $\tilde{z} \in U_{\widetilde{x}}^{c}$;

(3) $\tilde{\gamma}(\tilde{x}, 1)=\tilde{y}$; and

(4) $\tilde{\gamma}(\widetilde{z}, t) \in \widetilde{C}(\widetilde{z})$, for all $\widetilde{z} \in U_{\widetilde{x}}^{c}$ and $t \in[0,1]$.

Proof. Just take $\tilde{\gamma}=\Pi_{\tilde{x}}^{s u} \circ \widetilde{\Gamma}$ where $\Pi_{\tilde{x}}^{s u}$ and $\widetilde{\Gamma}$ are the maps defined in (2.2) and (2.5), respectively. See also [29]).

Remark 2.8. Notice that projecting by $\pi: \widetilde{M} \rightarrow M$ we have a similar result: given $x$ in $M$ and $\widetilde{x} \in \widetilde{M}$ such that $\pi(\widetilde{x})=x$ and $y \in \pi(\widetilde{C}(\widetilde{x}))$ then there are neighborhoods $U_{x}^{c}$ and $U_{y}^{c}$ in $\mathscr{F}^{c}(x)$ and a continuous map $\gamma: U_{x}^{c} \times[0,1] \rightarrow \mathscr{F}^{c}(x)$ such that

(1) $\gamma(z, 0)=z$ for all $z \in U_{x}^{c}$;

(2) $\gamma(z, 1) \in U_{y}^{c}$ for all $z \in U_{x}^{c}$;

(3) $\gamma(x, 1)=y$; and

(4) $\gamma(z, t) \in C(z)$, for all $z \in U_{x}^{c}$ and $t \in[0,1]$.

Corollary 2.9. $\widetilde{C}(x)$ is connected and arc-connected.

Recall that an accessibility class $A C(x)$ is trivial if $A C(x) \cap \mathscr{F}^{c}(x)$ is totally disconnected. Notice that by the map $\Gamma$ defined in (2.4) this does not depend on $x$, just on the accessibility class.

Lemma 2.10. The accessibility class $A C(x)$ is trivial if and only if $\widetilde{C}(\widetilde{x})=\{\widetilde{x}\}$ where $\pi(\tilde{x})=x$.

Proof. If $\widetilde{C}(\widetilde{x}) \neq\{\widetilde{x}\}$ then, since it is connected and arc-connected we have that $\pi(\widetilde{C}(\tilde{x})) \subset C(x)$ contains a non trivial connected set and so $\underset{\widetilde{F}}{C}(x)$ is not totally disconnected. On the other hand, if $\widetilde{C}(\tilde{x})=\{\tilde{x}\}$ then $\widetilde{A C}(\tilde{x}) \cap \widetilde{F}^{c}(\tilde{y})$ consists of a single point for any $\tilde{y}$ and in particular for those $\tilde{y}$ with $\pi(\tilde{y})=x$. Therefore $C(x)$ is at most countable and so totally disconnected. 
Corollary 2.11. The set $\{x \in M: A C(x)$ is nontrivial $\}$ is open in $M$.

Proof. Let $x \in M$ be such that $A C(x)$ is nontrivial and let $\tilde{x}$ such that $\pi(\tilde{x})=x$. Then $\widetilde{C}(\widetilde{x}) \neq\{\widetilde{x}\}$. Now, from Lemma 2.7 we get for any $\widetilde{z} \in \widetilde{F}^{c}(\widetilde{x})$ close enough to $\tilde{x}$ that $\widetilde{C}(\widetilde{z}) \neq\{\widetilde{z}\}$ and the lemma follows.

The above says that if we have a nontrivial accessibility class then nearby the classes are nontrivial. We will prove that the same holds when we perturb $f$ as well. We need the following lemma.

Lemma 2.12. Let $f \in \mathcal{E}^{r}$. Consider $x \in M$ and let $y \in A C(x)$. Then, there exist $U_{x}, U_{y}$ neighborhood of $x$ and $y$ respectively and $a C^{1}$ neighborhood of $f$ in $\varepsilon^{r}$ such that for any $g \in \mathcal{U}(f)$ and any $z \in U_{x}$ it holds that $A C(z) \cap U_{y} \neq \varnothing$.

Proof. Fix a su-path from $x$ to $y$ and fix also a small neighborhood $U_{y}$. As in the construction of the map $\Gamma$, there exists neighborhoods $U_{x}^{c}$ and $U_{y}^{c} \subset U_{y}$ such that any $z \in U_{x}^{c}$ can be joined by a su-path to a point in $U_{y}^{c}$. By the continuity of the stable and unstable manifolds in compact sets (with respect to $f$ ) and the continuous variation of the center foliation, there exists a $C^{1}$ neighborhood $U(f)$ such that for any $g \in \mathcal{U}(f)$ there exists a neighborhood $U_{x, g}^{c}$ in $\mathscr{F}^{c}(x, g)$ so that any $z \in U_{x, g}^{c}$ can be joined by a $s u$-path to a point in a neighborhood $U_{y, g}^{c} \subset \mathcal{F}^{c}(y, g)$. We may consider that $U_{y, g}^{c}$ is contained in $U_{y}$ for any $g \in U(f)$. Moreover, we can find a neighborhood $U_{x}$ so that for any $g \in U(f)$ and any $z \in U_{x}$ there is a $s u$-path joining $z$ with a point in $U_{x, g}^{c}$.

As a consequence, we have that a non-trivial accessibility class can not be destroyed by perturbations.

Corollary 2.13. Let $f \in \mathcal{E}^{r}$ and let $x \in M$ be such that $A C(x)$ is nontrivial. Then, there exist a neighborhood $U_{x}$ of $x$ and a neighborhood $U(f)$ in $\mathcal{E}^{r}$ (which can be considered $C^{1}$ open as well) such that for any $g \in U(f)$ and $z \in U_{x}$ the accessibility class $A C(z, g)$ is nontrivial.

Proof. Let $\tilde{x}$ be such that $\pi(\tilde{x})=x$. Since $A C(x)$ is nontrivial, then $\widetilde{C}(\tilde{x}) \neq\{\tilde{x}\}$. Let $\tilde{y} \in \widetilde{C}(\tilde{x}), \tilde{y} \neq \tilde{x}$. We have a su-path (in $\widetilde{M}$ ) joining $\tilde{x}$ to $\tilde{y}$. By Lemma 2.12 applied to the lift, there are disjoint open sets $U_{\tilde{x}}$ and $U_{\tilde{y}}$ of $\tilde{x}$ and $\tilde{y}$ in $\widetilde{M}$ and an open set $U(f)$ such that for any $g$ in $U(f)$ we have that any point in $U_{\tilde{x}}$ can be joined by $s u$ path of $\widetilde{g}$ with a point in $U_{\tilde{y}}$. And moreover, if we consider $\Pi_{\widetilde{g}}^{s u}: \widetilde{M} \rightarrow \mathscr{F}^{c}(\widetilde{x}, \widetilde{g})$, then $\Pi_{\tilde{g}}^{s u}\left(U_{\tilde{x}}\right)$ and $\Pi_{\tilde{g}}^{s u}\left(U_{\tilde{y}}\right)$ are open sets (in $\left.\mathscr{F}^{c}(\tilde{x}, \widetilde{g})\right)$ and disjoint. The result thus follows, since for any $\widetilde{z} \in \Pi_{\widetilde{g}}^{s u}\left(U_{\tilde{x}}\right)$ there is point in $\Pi_{\widetilde{g}}^{s u}\left(U_{\tilde{y}}\right)$ that belongs to $\widetilde{C}(\widetilde{z}, g)$.

Lemma 2.14. Let $x \in M$ and let $\tilde{x} \in \widetilde{M}$ with $\pi(\tilde{x})=x$. Let $\widetilde{\mathcal{F}}_{1}^{c}$ and $\widetilde{\mathcal{F}}_{2}^{c}$ be such that $\pi\left(\widetilde{\mathcal{F}}_{i}^{c}\right)=\mathscr{F}^{c}(x)$ and let ${\widetilde{C_{i}}}_{i}=\widetilde{A C}(\widetilde{x}) \cap \widetilde{\mathscr{F}}_{i}^{c}, i=1,2$. Then either $\pi\left(\mathcal{C}_{1}\right)$ and $\pi\left(\bigodot_{2}\right)$ are equal or disjoint. 
Proof. Assume that $\pi\left(\widetilde{C}_{1}\right) \cap \pi\left(\widetilde{C}_{2}\right) \neq \emptyset$. Let $z$ be in this intersection and let $\tilde{z}_{i} \in \widetilde{\mathscr{C}}_{i}$ be such that $\pi\left(\widetilde{z}_{i}\right)=z$. It follows that $\widetilde{C}_{i}=\widetilde{C}\left(\widetilde{z}_{i}\right)$. Let $\beta$ be a covering map, $\beta\left(\widetilde{z}_{1}\right)=\widetilde{z}_{2}$. Since $\beta$ sends $s u$-path in $\widetilde{M}$ to $s u$-path we conclude that $\beta\left(\widetilde{\mathscr{C}}_{1}\right) \subset \widetilde{\mathscr{C}}_{2}$ and $\beta^{-1}\left(\widetilde{\mathscr{e}}_{2}\right) \subset \widetilde{\mathscr{e}}_{1}$. Hence $\pi\left(\widetilde{\mathscr{e}}_{1}\right)=\pi\left(\widetilde{\mathscr{C}}_{2}\right)$.

Corollary 2.15. Let $x \in M$ and $\tilde{x} \in \widetilde{M}$ such that $\pi(\tilde{x})=x$. If $C(x)$ is open then $\pi(\widetilde{C}(\widetilde{x}))$ is the connected component of $C(x)$ that contains $x$.

Proof. It is a direct consequence of Lemmas 2.1, 2.3, and 2.14.

We now investigate the structure of accessibility classes when the center bundle has dimension two, that is $f \in \mathcal{E}^{r}$ and $\operatorname{dim} E^{c}=2$. The following important result is essentially contained in [29].

Theorem 2.16. Let $f \in \mathcal{E}^{r}$ and assume that $\operatorname{dim} E^{c}=2$. Let $\tilde{x} \in \widetilde{M}$. Then one and only one of the following holds:

(1) $\widetilde{C}(\widetilde{x})$ is open;

(2) $\widetilde{C}(\widetilde{x})=\{\widetilde{x}\}$;

(3) $\widetilde{C}(\widetilde{x})$ is a $C^{1}$ one dimensional manifold without boundary.

Proof. The same proof in [29, Proposition 5.2] yields that $\widetilde{C}(\widetilde{x})$ is either open, consists just of $\tilde{x}$ or it is a topological one dimensional manifold. Now, in case $\widetilde{C}(\widetilde{x})$ is a topological one dimensional manifold, by the $C^{1}$ homogeneity of $\widetilde{C}(\widetilde{x})$ and the result in [33] which says that a locally compact and $C^{1}$ homogeneous subset of a riemannian manifold is a $C^{1}$ submanifold, one get that in fact $\widetilde{C}(\widetilde{x})$ is of class $C^{1}$ (and without boundary).

Let us denote by $C_{0}(x)$ the arc-connected component of $C(x)$ that contains $x$. We remark that when $C(x)$ is open, then $C_{0}(x)$ is just the connected component of $C(x)$ that contains $x$.

Corollary 2.17. Let $f \in \mathcal{E}^{r}$ and assume that $\operatorname{dim} E^{c}=2$. Let $x \in M$ and $\tilde{x} \in \widetilde{M}$ with $\pi(\widetilde{x})=x$. Then $\pi(\widetilde{C}(\widetilde{x}))=C_{0}(x)$.

Proof. When $\widetilde{C}(x)$ is open or trivial then the result follows immediately. Thus, we just have to check it when $\widetilde{C}(x)$ is a one dimensional submanifold (without boundary).

From Lemma 2.1 and Corollary 2.9 we have $\pi(\widetilde{C}(\widetilde{x})) \subset C_{0}(x)$. On the other hand, let $\beta$ be an arc in $C_{0}(x)$ starting at $x$ and assume that it is not contained in $\pi(\widetilde{C}(\widetilde{x}))$. Let $t_{0}=\sup \{t: \beta([0, t]) \subset \pi(\widetilde{C}(\widetilde{x}))\}$. Let $y=\beta\left(t_{0}\right)$ (that belongs to $\pi(\widetilde{C}(\widetilde{x}))$ ). Since $\widetilde{C}(\widetilde{x})$ has no boundary, we have an arc $\alpha$ inside $\pi(\widetilde{C}(x))$ having $y$ in its interior, say joining $x_{1}$ with $x_{2}$. Now, applying Lemma 2.7 (or Remark 2.8) we have a continuous map $\gamma: U_{x_{1}}^{c} \times[0,1] \rightarrow \mathscr{F}^{c}(x)$ such that $\gamma\left(x_{1}, t\right)=\alpha(t)$ and that $\gamma(z, t) \in C(z)$ for any $t$ and $z \in U_{x_{1}}$. Then we conclude that there is an open set $U \subset U_{x_{1}}$ such that $\gamma(U \times[0,1]) \cap \beta \neq \emptyset$ (see Figure 2). This implies that $C(x)$ is open, a contradiction. Therefore, $C_{0}(x) \subset \pi(\widetilde{C}(\widetilde{x}))$. The proof is finished. 


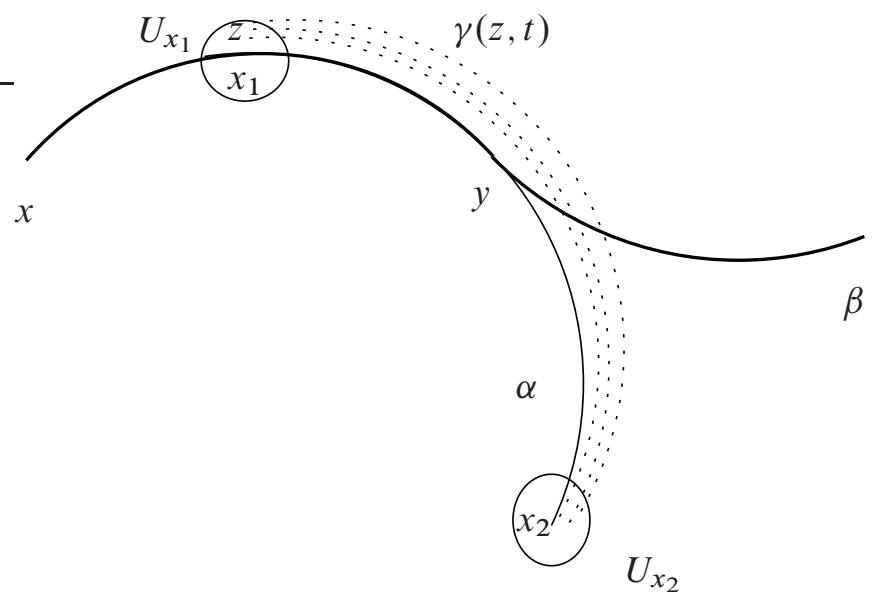

Figure 2. Proof of Corollary 2.17.

Next, we want to show that the set of one dimensional accessibility classes form a lamination. Let's recall the definition of a lamination (we state this for the case of lamination of subsets of a surface for simplicity).

Definition 2.18. Let $S$ be a surface and let $K \subset S$ be closed. We say that $K$ admits a $C^{1}$ lamination if $K$ has a partition into $C^{1}$ one dimensional manifolds (called leaves of the lamination) such that every point in $K$ has a neighborhood $U$ homeomorphic to $(-1,1) \times(-1,1)$ (called charts of the lamination) such that $K \cap U$ correspond to $F \times(-1,1)$, where $F$ is a closed set in $(-1,1)$ and every $\{f\} \times(-1,1)$, for $f \in F$, is inside a leaf of $K$, and tangent spaces of the leaves vary continuously.

Fix a center leaf $\widetilde{\mathscr{F}}^{c}$. Let $\widetilde{K}$ be the union of accessibility classes in $\widetilde{\mathscr{F}}^{c}$ which are $C^{1}$ one-dimensional submanifolds. Then $\widetilde{K}$ is partitioned by the accessibility classes $\widetilde{C}(\widetilde{x}), \widetilde{x} \in \widetilde{K}$. We want to prove that this partition form a $C^{1}$ lamination.

First we prove that the accessibility classes vary continuously in the $C^{1}$ topology:

Proposition 2.19. For $\widetilde{x} \in \widetilde{K}$, the curves $\widetilde{C}(\widetilde{x})$ vary continuously in the $C^{1}$ topology.

Before proving the proposition we need an elementary lemma from calculus (whose proof is left to the reader). Let us introduce some notations. Let $\gamma:[0,1] \rightarrow S$ be an $C^{1}$ arc of a surface $S$ and consider an $\varepsilon$ tubular neighborhood $N_{\gamma}$. This tubular neighborhood is diffeomorphic to $[0,1] \times[-\varepsilon, \varepsilon]$. Given a point in $N_{\gamma}$ we identify with coordinates $(t, s), t \in[0,1]$ and $s \in[-\varepsilon, \varepsilon]$.

We call the left side of $N_{\gamma}$ the boundary $\{0\} \times[-\varepsilon, \varepsilon]$ and right side the boundary $\{1\} \times[-\varepsilon, \varepsilon]$. We denote by $\xi: N_{\gamma} \rightarrow \gamma$ the orthogonal projection, i.e. in local coordinates $\xi(t, s)=t$.

Lemma 2.20. With the notations above, given $\delta>0$, there exists $\varepsilon=\varepsilon(\delta)$ such that if $\beta$ is a $C^{1}$ curve in $N_{\gamma}$ from the left to the right side (and do not intersect $\gamma$ ) then there is some $(t, s)=\beta(\widetilde{t})$ such that the angle $\angle(\dot{\beta}(\tilde{t}), \dot{\gamma}(t))<\delta$. 
Now we are ready to give a proof of Proposition 2.19. Since we will be working in a neighborhood of $\widetilde{F}^{c}$ we may assume that we are in trivialization chart of the tangent bundle $T \widetilde{\mathscr{F}}^{c}$, and so we may compare angles and norms of vectors in different tangent spaces.

Proof of Proposition 2.19. We need to prove that if $\widetilde{x} \in \widetilde{K}$ and $\widetilde{x}_{n} \in \widetilde{K}$ converges to $\tilde{x}$ then $T_{\widetilde{x}_{n}} \widetilde{e}\left(\widetilde{x}_{n}\right)$ converges to $T_{\widetilde{x}} \widetilde{e}(\widetilde{x})$. Assume that it is not true. Then there exists some sequence $\widetilde{x}_{n} \in \widetilde{K}$ converging to some point $\tilde{x} \in \widetilde{K}$ and some $\eta>0$ such that the angle $\angle\left(T_{\widetilde{x}_{n}} \widetilde{e}\left(\widetilde{x}_{n}\right), T_{\widetilde{x}} \widetilde{e}(\widetilde{x})\right)>\eta$, for all $n$.

Let $\tilde{y} \in \tilde{\mathcal{C}}(\tilde{x}), \tilde{y} \neq \tilde{x}$ and let $\Upsilon^{s u}$ a $s u$-path joining $\tilde{y}$ to $\tilde{x}$. We may consider an arc of $s u$-paths, i.e. for each $t \in[0,1]$ a su-path $\Upsilon_{t}^{s u}$ that vary continuously joining $\tilde{x}$ with some point $\Upsilon_{t}^{s u}(1) \in \widetilde{\mathcal{E}}(\tilde{x})$ such that $\Upsilon_{0}^{s u}$ is the trivial su-path and $\Upsilon_{1}^{s u}=\Upsilon^{s u}$. We may assume that the path $\Upsilon_{t}^{s u}(1)$ is the arc joining $\tilde{x}$ and $\tilde{y}$ in $\widetilde{e}(\tilde{x})$ denoted by $[\tilde{x}, \tilde{y}]$. We will consider tubular neighborhood $N$ of the $\operatorname{arc}[\tilde{x}, \tilde{y}]$.

The path $\Upsilon_{t}^{s u}$ allows us to consider (see the last item of properties of the map $\Gamma$ in (2.4) and equivalent for (2.5)) a map $\phi_{t}: B\left(\widetilde{x}, r_{t}\right) \rightarrow \widetilde{F}^{c}$ which is a $C^{1}$ diffeomorphism onto its image that contains $\Upsilon_{t}^{s u}(1)$. We may choose $r_{t}=r$ independent of $t$. The family $\phi_{t}$ varies continuously in the $C^{1}$ topology due to the local holonomy is $C^{1}$ inside center stable and center unstable leaves, the center leaves vary continuously in the $C^{1}$ topology and the path $\Upsilon_{t}^{s u}$ varies continuously with $t$.

Given $\theta>0$ there exists $\delta_{0}>0$ and $\rho_{0}>0$ such that for any $t$ if $\operatorname{dist}(\widetilde{z}, \tilde{x})<\rho_{0}$ and $\angle\left(T_{\tilde{x}} \widetilde{e}(\widetilde{x}), w\right)>\theta$ then the angle

$$
\angle\left(d\left(\phi_{t}\right)_{\tilde{x}}\left(T_{\tilde{x}} \widetilde{e}(\widetilde{x})\right), d\left(\phi_{t}\right)_{\tilde{z}}(w)\right)=\angle\left(T_{\phi_{t}(\widetilde{x})} \widetilde{e}(\widetilde{x}), d\left(\phi_{t}\right)_{\tilde{z}}(w)\right)>\delta_{0} .
$$

On the other hand, given $\delta_{1}>0$ there exists $\varepsilon_{1}>0$ such that if

$$
\operatorname{dist}\left(\xi\left(\phi_{t}(\widetilde{z})\right), \phi_{t}(\widetilde{x})\right)<\varepsilon_{1} \text { then } \angle\left(T_{\xi\left(\phi_{t}(\widetilde{z})\right)} \widetilde{e}(\widetilde{x}), T_{\phi_{t}(\widetilde{x})} \widetilde{e}(\widetilde{x})\right)<\delta_{1} \text {. }
$$

Notice also that there exists $\rho>0$ such that for any $t$ if

$$
\operatorname{dist}(\tilde{x}, \widetilde{z})<\rho \text { then } \operatorname{dist}\left(\xi\left(\phi_{t}(\widetilde{z})\right), \phi_{t}(\widetilde{x})\right)<\varepsilon_{1} .
$$

Consider $\hat{x}$ and $\hat{y}$ in $\widetilde{e}(\widetilde{x})$ between $\tilde{x}$ and $\tilde{y}$. Denote by $\gamma=[\hat{x}, \hat{y}]$ the $\operatorname{arc}$ in $\widetilde{e}(\widetilde{x})$ joining $\hat{x}$ and $\hat{y}$.

Let $\theta=\eta$ and take $\delta_{0}=\delta_{0}(\theta)$ from (2.6). Choose $\delta_{1}>0$ such that $\delta_{0}-\delta_{1}=$ $\delta>0$ and let $\varepsilon_{1}$ from (2.7). Choose $\rho<\rho_{0}$ such that (2.8) holds.

For this $\delta$ choose an $\varepsilon$ tubular neighborhood $N_{[\hat{x}, \hat{y}]}$ as in Lemma 2.20 and such that $N_{[\hat{x}, \hat{y}]} \subset \cup_{t \in[0,1]} \phi_{t}(B(\tilde{x}, \rho))$. 
Now, if $\tilde{x}_{n}$ is close enough to $\tilde{x}$ then $\beta_{n}(t)=\phi_{t}\left(\tilde{x}_{n}\right), t \in[0,1]$ is a curve that crosses the $N_{[\hat{x}, \hat{y}]}$ from the left to the right side. On the other hand, if $t$ is such that $\beta_{n}(t) \in N_{[\hat{x}, \hat{y}]}$ then $\operatorname{span}\left(\dot{\beta}_{n}\right)=d\left(\phi_{t}\left(\widetilde{x}_{n}\right)\right)\left(T_{\widetilde{x}_{n}} \widetilde{e}\left(\tilde{x}_{n}\right)\right)$ and so

$$
\begin{aligned}
\angle\left(\dot{\beta}_{n}(t), T_{\xi\left(\beta_{n}(t)\right)} \widetilde{e}(\tilde{x})\right)= & \angle\left(d \phi_{t}\left(\widetilde{x}_{n}\right) T_{\widetilde{x}_{n}} \widetilde{e}\left(\widetilde{x}_{n}\right), T_{\xi\left(\phi_{t}\left(\widetilde{x}_{n}\right)\right)} \widetilde{e}\left(\widetilde{x}_{n}\right)\right) \\
> & \angle\left(d \phi_{t}\left(\widetilde{x}_{n}\right) T_{\widetilde{x}_{n}} \widetilde{e}\left(\widetilde{x}_{n}\right), d \phi_{t}(\widetilde{x}) T_{\widetilde{x}} \widetilde{e}(\widetilde{x})\right. \\
& \quad-\angle\left(T_{\xi\left(\phi_{t}\left(\widetilde{x}_{n}\right)\right)} \widetilde{e}\left(\widetilde{x}_{n}\right), d \phi_{t}(\widetilde{x}) T_{\widetilde{x}} \widetilde{e}(\widetilde{x})\right) \\
> & \delta_{0}-\delta_{1}=\delta,
\end{aligned}
$$

which is a contradiction with Lemma 2.20 .

Corollary 2.21. Let $\widetilde{F}^{c}$ be a center leaf in $\widetilde{M}$ and assume that there is no trivial accessibility classes. Then the set $\widetilde{K}$ of non-open accessibility classes admits a $C^{1}$ lamination whose leaves are accessibility classes. The same holds for the set of non open accessibility classes $K$ in $\mathscr{F}^{c}$ whose leaves are connected components of accessibility classes $C_{0}(x)$ for $x \in K$.

Proof. Since there is no trivial accessibility class the set $\widetilde{K}$ is closed. From Proposition 2.19 and using transversal sections it is not difficult to construct a chart for each $\tilde{x} \in \widetilde{K}$.

\section{Perturbation lemmas}

In this section we prove our main perturbation techniques that allow us to prove our theorems. These are Lemmas 3.5 and 3.7. Before we state and prove these lemmas we need some elementary results, the first one says that some perturbations of the identity can be thought as translations in terms of local coordinates, no matter if we are in the conservative world, symplectic world, etc.

Let $M$ be a manifold of dimension $d$ and let $S \subset M$ be an embedded submanifold of $M$ of dimension $k$. Let $z \in S$ and let $U$ be a neighborhood of $z$ in $M$ and let $V$ be the connected component of $U \cap S$ containing $z$. We say that we have local canonical coordinates in $V$ if we have a coordinate chart (or parametrization) $\varphi: U \rightarrow \mathbb{R}^{d}$ and $\varphi(V)=V_{0} \subset \mathbb{R}^{k}$ with $\varphi(z)=0$.

It is a consequence of Darboux Theorem that if $\omega$ is a symplectic form in $M$ such that $\omega_{/ S}$ is symplectic and $k=2 j, d=2 l$ and we write coordinates in $\mathbb{R}^{k}$ as $\left(x_{1}, \ldots, x_{j}, y_{1}, \ldots, y_{j}\right)$ and in $\mathbb{R}^{d}$ as $\left(x_{1}, \ldots, x_{l}, y_{1}, \ldots, y_{l}\right)$ then we may assume that the local chart verifies $\varphi^{*}\left(\sum_{i=1}^{d} d x_{i} \wedge d y_{i}\right)=\omega \mid U$ and $\varphi^{*}\left(\sum_{i=1}^{j} d x_{i} \wedge d y_{i}\right)=$ $\omega \mid V$, see e.g. [24] and [36].

And in case $m$ is a volume form it is well known that we can choose local coordinates in $\mathbb{R}^{d}$ as $\left(x_{1}, \ldots, x_{d}\right)$, we may assume also that $\varphi^{*}\left(d x_{1} \wedge \cdots \wedge d x_{d}\right)=m$, i.e. in local coordinates the volume form is the standard volume form in $\mathbb{R}^{d}$ (see e.g. [24]). 
The next lemma says that we can glue an arbitrary small translation near a point with the identity outside a neighborhood in the conservative and symplectic setting. Sophisticated versions of this problem can be found in [13] and [2] (pasting lemma).

Lemma 3.1. Let $M$ be a $C^{r}$ manifold and let $S \subset M$ be a $C^{r}$ submanifold of $M, r \geq 1$. Let $z \in S$ and let $U$ be a given neighborhood of $z$ in $M$ such that $V:=S \cap U$ has local canonical coordinates. Then, there exist $V^{\prime}, z \in V^{\prime} \subset V \subset U$ and $\varepsilon_{0}>0$ such that for any $0 \leq \varepsilon \leq \varepsilon_{0}$ there exists $\delta>0$ such that for any $w \in \mathbb{R}^{k}$, $\|w\|<\delta$ there exists a diffeomorphism $h: M \rightarrow M$ satisfying:

(1) $h$ is $\varepsilon-C^{r}$ close to identity;

(2) $h \equiv$ id on $U^{c}$;

(3) $h$ preserves $V$, i.e. $h(V)=V$;

(4) $h \mid V^{\prime}$ in local coordinates is given by

$$
y \mapsto h(y)=y+w .
$$

If $\omega$ is a symplectic form in $M$ and $\omega / S$ is symplectic then $h$ can be taken to be a symplectomorphism. If $m$ is a volume form in $M$, we can take $h$ to preserves the volume form $m$.

Proof. In the general case (i.e neither conservative nor symplectic) the solution is easy, just take the time $t$ map (with $t$ small enough) of the flow generated by a vector field $X$ (in the local coordinates) such that $X(x)=v$ for $v \in \mathbb{R}^{k},\|v\|=1$ and it is identically zero outside a neighborhood of the origin. The same idea works in the conservative setting taking $X$ to be divergence free vector field and in the symplectic setting taking a Hamiltonian vector field.

Let's consider the conservative setting. Recall that we have local coordinates, i.e. a chart $\varphi: U \rightarrow \mathbb{R}^{d}$ and $\varphi(V)=V_{0} \subset \mathbb{R}^{k}$ with $\varphi(z)=0$ and $\varphi^{*}\left(d x_{1} \wedge \cdots \wedge d x_{d}\right)=m$. Let $\psi: \mathbb{R}^{d} \rightarrow \mathbb{R}$ be a bump function such that it is equal to 1 in a neighborhood of 0 and it is identically zero outside a neighborhood of 0 as well (with closure contained in $\varphi(U)$ ). Let $w \in \mathbb{R}^{k},\|w\|=1$. By a linear change of coordinates (preserving $\mathbb{R}^{k}$ ) we may assume that $w=e_{1}$. Consider the function $\chi: \mathbb{R}^{d} \rightarrow \mathbb{R}$ given by

$$
\chi(x)=\chi\left(x_{1}, x_{2}, \ldots, x_{d}\right)=\psi(x) x_{2} .
$$

Then, taking $X(x)=\left(\frac{\partial \chi}{\partial x_{2}},-\frac{\partial \chi}{\partial x_{1}}, 0, \ldots, 0\right)$ we have that $X$ is divergence free. Now, taking the time $t$ map of the flow generated by $X$ for $t$ small we get the lemma.

Let's consider the symplectic case. We may assume without loss of generality that $U$ is contained in a tubular neighborhood of $S$ and $U=U^{\prime} \times D, D$ fibers of the tubular neighborhood. Choose open balls $V_{1} \subset V_{1}^{\prime} \subset U_{1} \subset U_{1}^{\prime} \subset U$ centered in $z$ and a $C^{\infty}$ bump function $\psi: M \rightarrow \mathbb{R}$ so that $\psi \mid U_{1}^{c} \equiv 0$ and $\psi \mid V_{1} \equiv 1$. 
For simplicity we will assume that $S$ is bidimensional. Let $u=\left(u_{1}, u_{2}\right)$ be a unit vector in $\mathbb{R}^{2}$ and let $H_{0}^{u}: \mathbb{R}^{2} \rightarrow \mathbb{R}$ be defined by $H_{0}^{u}(x, y)=y u_{1}-x u_{2}$. Notice that $X_{H_{0}^{2}}=u$ in $\mathbb{R}^{2}$.

Let $\widetilde{H}_{0}^{u}: \mathbb{R}^{2} \rightarrow \mathbb{R}$ be $\widetilde{H}_{0}^{u}=\psi \cdot H_{0}^{u}$ and let $H_{1}^{u}: U \rightarrow \mathbb{R}$ be $H_{1}^{u}(y)=$ $H_{0}^{u}(\varphi(\pi(y)))$, where $\pi$ is the projection along the fibers of the tubular neighborhood, and let $H^{u}: M \rightarrow \mathbb{R}$ be such that

- $H^{u} \equiv 0$ in $U^{c}$, and

- $H^{u}=\psi(y) H_{1}^{u}(y)$ if $y \in U$.

Notice that $H^{u}$ is $C^{\infty}$ and the $C^{r}$ norm is bounded by a constant $K$ that does not depend on $u$.

Let $y \in S \cap V_{1}$. We claim that $X_{H^{u}}(y)=X_{H^{u}}^{S}(y)$, where $X_{H}^{S}$ is the hamiltonian field of $H \mid S$. Indeed, $X_{H^{u}}(y)$ is defined as $\omega\left(X_{H^{u}}(y), \cdot\right)=-d H_{y}^{u}$ and $X_{H^{u}}^{S}(y)$ as $\omega_{\mid S}\left(X_{H^{u}}^{S}(y), \cdot\right)=-d\left(H^{u} \mid S\right)_{y}$. For $y \in V_{1} \cap S, H^{u}(y)=H^{u}(\pi(y))$ and so $d H^{u}=d H^{u} \mid S \circ d \pi$ and hence $d H_{y}^{u} \mid\left(T_{y} S\right)^{\perp \omega}=0$. Thus, for any $v \in$ $\left(T_{y} S\right)^{\perp_{\omega}}, \omega\left(X_{H^{u}}(y), v\right)=0$, then $X_{H^{u}}(y) \in T_{y} S$ and so, since for any $w \in T_{y} S$, we have

$$
\omega\left(X_{H^{u}}(y), w\right)=\omega_{\mid S}\left(X_{H^{u}}^{S}(y), w\right) .
$$

We conclude that

$$
X_{H^{u}}(y)=X_{H^{u}}^{S}(y) .
$$

This proves the claim. Finally, taking the time $t$ map of the corresponding hamiltonian flow, for $t$ small enough, we conclude the lemma.

For $f \in \mathcal{E}^{r}$ we denote the stable manifold of size $\varepsilon$ of a center leaf $\widetilde{F}_{1}^{c}$ by $W_{\varepsilon}^{s}\left(\mathcal{F}_{1}^{c}\right):=\bigcup_{z \in \mathcal{F}_{1}^{c}}\left(W_{\varepsilon}^{s}(z)\right)$.

Remark 3.2. If $\mathscr{F}_{1}^{c}$ is a compact periodic center leaf and $w \in W_{\varepsilon}^{s}\left(\mathcal{F}_{1}^{c}\right)$ then there exists $\varepsilon_{0}$ such that $w \in W_{\varepsilon_{0}}^{s}\left(\widetilde{F}_{1}^{c}\right)$ but $w \notin \overline{f^{n}\left(W_{\varepsilon_{0}}^{s}\left(\widetilde{F}_{1}^{c}\right)\right)}$, for all $n \geq 1$.

Lemma 3.3. Let $f \in \mathcal{E}^{r}$ and let $\mathscr{F}_{1}^{c}$ be a compact periodic center leaf of $f$. Let $x \in \widetilde{F}_{1}^{c}$ and let $B$ be a small neighborhood of $x$ in $M$. Then there exist $p_{1}, p_{2}, w_{1}, w_{2}, z_{1}, z_{2} \in B, \varepsilon_{0}, \varepsilon_{1}, \varepsilon_{2}>0$, and $U_{1}, U_{2}$ disjoint neighborhoods of $w_{1}, w_{2}$ in $M$ such that, for $i=1,2$,

(1) $\mathscr{F}^{c}\left(p_{i}\right), i=1,2$, are periodic compact center leaves;

(2) $w_{i} \in W_{\varepsilon_{0}}^{s}(x) \cap W_{\varepsilon_{i}}^{u}\left(p_{i}\right)$;

(3) $z_{i} \in W_{\varepsilon_{0}}^{u}\left(\widetilde{F}_{1}^{c}\right) \cap W_{\varepsilon_{i}}^{s}\left(p_{i}\right)$;

(4) $U_{i} \cap f^{n}\left(W_{\varepsilon_{0}}^{s}\left(\mathcal{F}_{1}^{c}\right)\right)=\emptyset$, for all $n \geq 1$;

(5) $U_{i} \cap f^{-n}\left(W_{\varepsilon_{i}}^{u}\left(\mathscr{F}^{c}\left(p_{i}\right)\right)\right)=\emptyset$, for all $n \geq 1$;

(6) $U_{i} \cap f^{n}\left(W_{\varepsilon_{i}}^{s}\left(\mathcal{F}^{c}\left(p_{j}\right)\right)\right)=\emptyset$, for all $n \geq 0$ and $i, j=1,2$; and

(7) $U_{i} \cap f^{-n}\left(W_{\varepsilon_{0}}^{u}\left(\mathcal{F}_{1}^{c}\right)\right)=\emptyset$, for all $n \geq 0$. 
Proof. Let $B_{0}, x \in B_{0} \subset B$ be a foliated chart of the center foliation:

$$
\varphi: B_{0} \rightarrow D^{m-k} \times D^{k},
$$

where $D^{m-k}$ is a disk in $\mathbb{R}^{m-k}$ and $D^{k}$ is a disk in $\mathbb{R}^{k}$, and the center foliation in $B_{0}$ through $\varphi$ is $\{y\} \times D^{k}$ and $\varphi(x)=(0,0)$.

Let $P_{x}$ be the plaque of $x$ in $B_{0}$. We may assume that $\mathscr{F}_{1}^{c} \cap B_{0}=P_{x}$. In $B_{0}$, we identify point in the same plaque, $B_{0} / \sim \cong D^{m-k}$. Let $P: B_{0} \rightarrow B_{0} / \sim$ the projection map.

For $z \in B_{0}$, denote by $\hat{z}:=P(z)$ the plaque of $z$ and denote by $W_{B_{0}}^{c *}(z)$, $*=s, u$, the connected component of $W_{\varepsilon}^{*}\left(F^{c}(z)\right) \cap B_{0}$ that contains $z$, and by $W_{B_{0}}^{*}(\hat{z}):=P\left(W^{c *}(z)\right)\left(B_{0}\right.$ is small compared to $\left.\varepsilon\right)$.

In a neighborhood $W$ of $\hat{x}$ we have local product structure. Since periodic compact center leaves are dense, we may choose $\hat{p}_{1} \in W$ such that $\hat{p}_{1}$ is contained in a compact periodic center leaf. (This compact center leaf may intersects $B_{0}$ in other plaques than $\hat{p}_{1}$, but if it does, intersects finitely many plaques in $B_{0}$ ).

Let $\hat{w}_{1}=W_{B_{0}}^{s}(\hat{x}) \cap W_{B_{0}}^{u}\left(\hat{p}_{1}\right)$ and $w_{1}=W_{\varepsilon}^{s}(x) \cap P^{-1}\left(\hat{w}_{1}\right), p_{1}=W_{\varepsilon}^{u}\left(w_{1}\right) \cap$ $P^{-1}\left(\hat{p}_{1}\right), \hat{z}_{1}=W_{B_{0}}^{u}(\hat{x}) \cap W_{B_{0}}^{s}\left(\hat{p}_{1}\right)$, and $z_{1}=W_{\varepsilon}^{s}\left(p_{1}\right) \cap P^{-1}\left(\hat{z}_{1}\right)$.
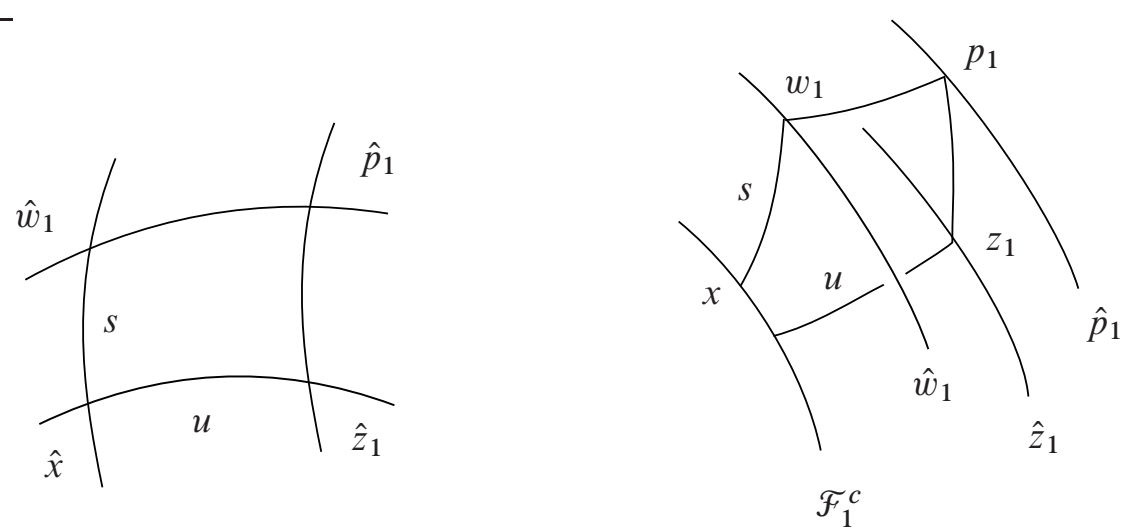

Figure 3. 4-legged path.

Now, we can find $\varepsilon_{0}, \varepsilon_{1}>0$ such that

- $w_{1} \in W_{\varepsilon_{0}}^{s}\left(\mathscr{F}_{1}^{c}\right)$ but $w_{1} \notin \overline{f^{n}\left(W_{\varepsilon_{0}}^{S}\left(\mathscr{F}_{1}^{c}\right)\right)}, n \geq 1$, and

- $w_{1} \in W_{\varepsilon_{1}}^{u}\left(\mathscr{F}^{c}\left(p_{1}\right)\right)$ but $w_{1} \notin \overline{f^{-n}\left(W_{\varepsilon_{1}}^{u}\left(\mathscr{F}^{c}\left(p_{1}\right)\right)\right)}, n \geq 1$.

Now, we may take $\hat{p}_{2}$ close to $\hat{p}_{1}$ and contained in a compact periodic leaf and such that, if we set $\hat{w}_{2}=W_{B_{0}}^{s}(\hat{x}) \cap W_{B_{0}}^{u}\left(\hat{p}_{2}\right), w_{2}=W_{\varepsilon}^{s}(x) \cap P^{-1}\left(\hat{w}_{2}\right), p_{2}=$ $W_{\varepsilon}^{u}\left(w_{2}\right) \cap P^{-1}\left(\hat{p}_{2}\right), \hat{z}_{2}=W_{B_{0}}^{u}(\hat{x}) \cap W_{B_{0}}^{s}\left(\hat{p}_{2}\right)$, and $z_{2}=W_{\varepsilon}^{s}\left(p_{2}\right) \cap P^{-1}\left(\hat{z}_{2}\right)$ then, $w_{2} \in W_{\varepsilon_{0}}^{s}\left(\mathscr{F}_{1}^{c}\right)$ but $w_{2} \notin \overline{f^{n}\left(W_{\varepsilon_{0}}^{s}\left(\widetilde{F}_{1}^{c}\right)\right)}$, for $n \geq 1$, if $\hat{p}_{2}$ is close enough to $\hat{p}_{1}$. Now, we may choose $\varepsilon_{2}>0$ such that $w_{2} \in W_{\varepsilon_{2}}^{u}\left(\mathscr{F}^{c}\left(p_{2}\right)\right)$ but $w_{2} \notin \overline{f^{-n}\left(W_{\varepsilon_{2}}^{u}\left(\mathscr{F}^{c}\left(p_{1}\right)\right)\right)}$, $n \geq 1$. 
Observe that trivially it holds that,

$$
\begin{array}{ll}
w_{i} \notin \overline{f^{n}\left(W_{\varepsilon}^{s}\left(\mathscr{F}^{c}\left(p_{j}\right)\right)\right)}, & n \geq 0, i, j=1,2 ; \text { and } \\
w_{i} \notin \overline{f^{-n}\left(W_{\varepsilon}^{u}\left(\mathscr{F}_{1}^{c}\right)\right)}, & n \geq 0, i=1,2 .
\end{array}
$$

From this it is easy to find the neighborhoods $U_{1}, U_{2}$. The proof is complete.

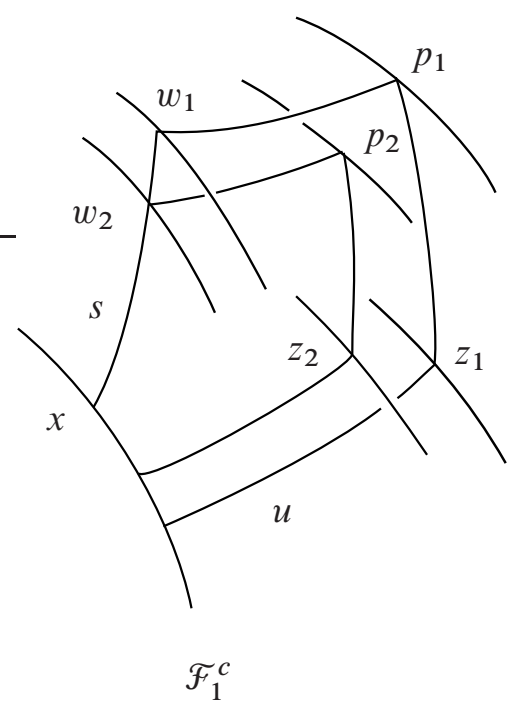

Figure 4. Lemma 3.3.

Given $f \in \mathcal{E}^{r}$ and $w \in W_{\varepsilon}^{s}(x)$ recall that we denote $\Pi_{f}^{s}\left(\mathscr{F}^{c}(x), \mathscr{F}^{c}(w)\right)$ the (local) holonomy map along the stable foliation in $\mathscr{F}^{c s}(x)$ from a neighborhood of $x$ in $\mathscr{F}^{c}(x)$ onto a neighborhood of $w$ in $\mathscr{F}^{c}(w)$. For the sake of simplicity we set $\hat{x}=\mathscr{F}^{c}(x), \hat{w}=\mathscr{F}^{c}(w)$ and $\Pi_{f}^{s}(\hat{x}, \hat{w})=\Pi_{f}^{s}\left(\mathscr{F}^{c}(x), \mathscr{F}^{c}(w)\right)$. Moreover, if $w \in \mathscr{F}^{c s}(x)$ (although perhaps $w \notin W^{s}(x)$ ) we still set $\Pi_{f}^{s}(\hat{x}, \hat{w})$ the local holonomy map form a neighborhood of $x$ in $\hat{x}=\mathscr{F}^{c}(x)$ onto a neighborhood of a point in $W^{s}(x) \cap \mathscr{F}^{c}(w)$ of $\hat{w}=\mathscr{F}^{c}(w)$ along the stable foliation where the point in $W^{s}(x) \cap \mathscr{F}^{c}(w)$ should be clear from the context. Analogous remark and notation holds for $\Pi_{f}^{u}$.

Remark 3.4. In the setting of Lemma 3.3 notice that $\Pi_{f}^{u}\left(\hat{z}_{i}, \hat{x}\right)\left(z_{i}\right)$ belongs to $C_{0}(x)$. Moreover, if $h: M \rightarrow M$ is a diffeomorphism close to the identity such that $h \equiv \mathrm{id}$ 
in $\left(U_{1} \cup U_{2}\right)^{c}$ and preserves $V_{i}$ the connected component of $U_{i} \cup \mathcal{F}^{c}\left(w_{i}\right)$ that contains $w_{i}$ then if we define $g=f \circ h^{-1}$ we have:

$$
\left.\begin{array}{l}
\mathscr{F}^{c}(*, f)=\mathscr{F}^{c}(*, g) \quad \text { where } *=x, p_{1}, p_{2} ; \\
V_{i} \subset \mathscr{F}^{c}\left(w_{i}, g\right) ; \\
\mathcal{F}_{\text {loc }}^{c}\left(z_{i}, f\right)=\mathscr{F}_{\text {loc }}^{c}\left(z_{i}, g\right) ; \\
\Pi_{g}^{s}\left(\hat{w}_{i}, \hat{x}\right)=\Pi_{f}^{s}\left(\hat{w}_{i}, \hat{w}_{i}\right) \circ h ; \\
\Pi_{g}^{u}\left(\hat{w}_{i}, \hat{p}_{i}\right)=\Pi_{f}^{u}\left(\hat{w}_{i}, \hat{p}_{i}\right) ; \\
\Pi_{g}^{s}\left(\hat{z}_{i}, \hat{p}_{i}\right)=\Pi_{f}^{s}\left(\hat{z}_{i}, \hat{p}_{i}\right) ; \text { and } \\
\Pi_{g}^{u}\left(\hat{x}, \hat{p}_{i}\right)=\Pi_{f}^{u}\left(\hat{x}, \hat{p}_{i}\right) .
\end{array}\right\}
$$

Denote $\operatorname{supp}(f \neq g)=\{y: f(y) \neq g(y)\}$. The next lemma says that we can destroy trivial accessibility class.

Lemma 3.5. Let $\mathcal{E}$ be as in Theorem 2 and let $f \in \mathcal{E}$ and let $\mathcal{F}^{c}(x)$ be a periodic compact center leaf of $f$. Assume that $C_{0}(x)=\{x\}$. Then, there exists neighborhood $V_{x}$ of $x$ in $\mathscr{F}^{c}(x)$ and $\varepsilon_{0}>0$ such that for any $0<\varepsilon \leq \varepsilon_{0}$ there exists $g \in \mathcal{E}$, with $\operatorname{dist}_{C^{r}}(f, g)<\varepsilon$ such that:

(1) $\operatorname{supp}(f \neq g)$ is disjoint from the $f$-orbit of $\mathscr{F}^{c}(x)$,

(2) for any $y \in V_{x}$ we have $\left.C_{0}(y, g)\right) \neq\{y\}$.

Proof. Let $x, w_{1}, w_{2}, p_{1}, p_{2}, z_{1}, z_{2}$ and $U_{1}, U_{2}$ be as in Lemma 3.3. As before, we write $\hat{w}_{i}=\mathscr{F}^{c}\left(w_{i}, f\right), \hat{z}_{i}=\mathscr{F}^{c}\left(z_{i}, f\right)$, and $\hat{p}_{i}=\mathscr{F}^{c}\left(p_{i}, f\right)$.

Since $C_{0}(x, f)=\{x\}$, we have $\Pi_{f}^{u}\left(\hat{z}_{i}, \hat{x}\right)\left(z_{i}\right)=x$ and

$$
\Pi_{f}^{s}\left(\hat{w}_{i}, \hat{x}\right) \circ \Pi_{f}^{u}\left(\hat{p}_{i}, \hat{w}_{i}\right) \circ \Pi_{f}^{s}\left(\hat{z}_{i}, \hat{p}_{i}\right) \circ \Pi_{f}^{u}\left(\hat{x}, \hat{z}_{i}\right)(x)=x, \quad i=1,2 .
$$

Let $W_{0}$ be a small neighborhood of $x$ in $\mathscr{F}^{c}(x)$ and let $W_{1} \subset W_{0}$ such that $W_{1} \subset \Pi_{f}^{s}\left(\hat{w}_{i}, \hat{x}\right) \circ \Pi_{f}^{u}\left(\hat{p}_{i}, \hat{w}_{i}\right) \circ \Pi_{f}^{s}\left(\hat{z}_{i}, \hat{p}_{i}\right) \circ \Pi_{f}^{u}\left(\hat{x}, \hat{z}_{i}\right)\left(W_{0}\right)$.

We may assume that $U_{i}$ are small so that if $V_{i}$ is the connected component of $U_{i} \cap \hat{w}_{i}$ that contains $w_{i}$ then $\Pi_{f}^{s}\left(\hat{w}_{i}, \hat{x}\right)\left(V_{i}\right) \subset W_{1}$.

Let $\varepsilon>0$ be given (small) and let $V_{i}^{\prime}$ as in Lemma 3.1 for the submanifolds $\hat{w}_{i}$ corresponding to $V_{i}$ and let $V_{x} \subset \Pi_{f}^{s}\left(\hat{w}_{i}, \hat{x}\right)\left(V_{i}^{\prime}\right)$.

Let $l_{i}: V_{i} \rightarrow \hat{w}_{i}$ be defined by

$$
l_{i}=\Pi_{f}^{u}\left(\hat{p}_{i}, \hat{w}_{i}\right) \circ \Pi_{f}^{s}\left(\hat{z}_{i}, \hat{p}_{i}\right) \circ \Pi_{f}^{u}\left(\hat{x}, \hat{z}_{i}\right) \circ \Pi_{f}^{s}\left(\hat{w}_{i}, \hat{x}\right) .
$$

Note that $l_{i}$ is a $C^{1}$ map and

$\Pi_{f}^{s}\left(\hat{w}_{i}, \hat{x}\right) \circ \Pi_{f}^{u}\left(\hat{p}_{i}, \hat{w}_{i}\right) \circ \Pi_{f}^{s}\left(\hat{z}_{i}, \hat{p}_{i}\right) \circ \Pi_{f}^{u}\left(\hat{x}, \hat{z}_{i}\right)=\Pi_{f}^{s}\left(\hat{w}_{i}, \hat{x}\right) \circ l_{i} \circ\left(\Pi_{f}^{s}\left(\hat{w}_{i}, \hat{x}\right)\right)^{-1}$. 
Let's look $l_{1}$ in the local canonical coordinates and let $v$ in $\mathbb{R}^{k},\|v\|<\delta$ be such that $-v$ is a regular value of $l_{1}-$ id. For this $v$, choose $h_{1}: M \rightarrow M$ as in Lemma 3.1 (in the appropriate setting for $\mathcal{E}$ ) and so $h_{1} \circ l_{1}$ has finitely many fixed points in $\overline{V_{1}^{\prime}}$. Indeed, if $q$ is a fixed point in $\overline{V_{1}^{\prime}}$ of $h_{1} \circ l_{1}$ then $l_{1}(q)-q=-v$ and since $-v$ is a regular value of $l_{1}-$ id it is an isolated fixed point.

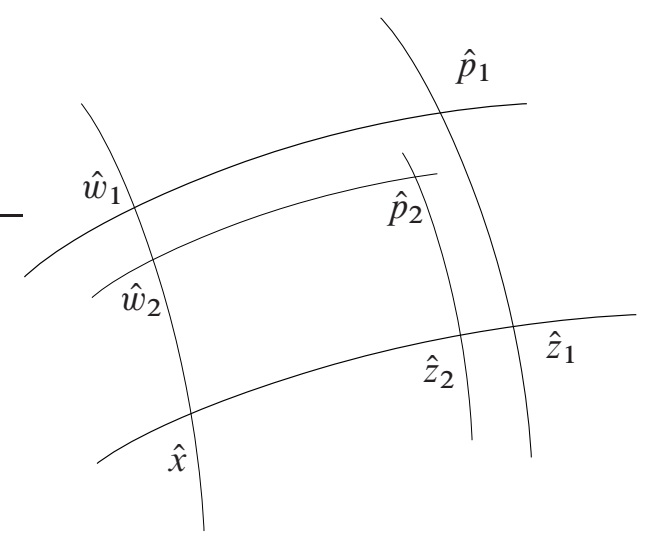

Figure 5. Perturbing trivial accessibility classes.

Let $q_{1}, \ldots, q_{\ell}$ be the projection of these fixed points in $V_{2}$, i.e. $\left\{q_{1}, \ldots, q_{\ell}\right\}$ $=\Pi_{f}^{s}\left(\hat{w}_{1}, \hat{w}_{2}\right)\left(\operatorname{Fix}\left(h_{1} \circ l_{1} \mid \bar{V}_{1}\right)\right) \cap V_{2}$. Choose $h_{2}: M \rightarrow M$ as in Lemma 3.1 (corresponding to $U_{2}, V_{2}$ ) such that no $q_{i}$ is fixed by $h_{2} \circ l_{2}$.

Let $g: M \rightarrow M$ be $g=f \circ h^{-1}$ where $h: M \rightarrow M$ is defined by

$$
h(z)= \begin{cases}h_{1}(z) & \text { if } z \in U_{1}, \\ h_{2}(z) & \text { if } z \in U_{2}, \\ z & \text { otherwise }\end{cases}
$$

It is not difficult to see that (see Remark 3.4):

$$
\begin{aligned}
\Pi_{g}^{s}\left(\hat{w}_{i}, \hat{x}\right) \circ \Pi_{g}^{u}\left(\hat{p}_{i}, \hat{w}_{i}\right) \circ \Pi_{g}^{s}\left(\hat{z}_{i}, \hat{p}_{i}\right) \circ \Pi_{g}^{u}\left(\hat{x}, \hat{z}_{i}\right) \\
=\Pi_{f}^{s}\left(\hat{w}_{i}, \hat{x}\right) \circ h_{i} \circ l_{i} \circ\left(\Pi_{f}^{s}\left(\hat{w}_{i}, \hat{x}\right)\right)^{-1} .
\end{aligned}
$$

Now, the maps

and

$$
\Pi_{g}^{s}\left(\hat{w}_{1}, \hat{x}\right) \circ \Pi_{g}^{u}\left(\hat{p}_{1}, \hat{w}_{1}\right) \circ \Pi_{g}^{s}\left(\hat{z}_{1}, \hat{p}_{1}\right) \circ \Pi_{g}^{u}\left(\hat{x}, \hat{z}_{1}\right)
$$

$$
\Pi_{g}^{s}\left(\hat{w}_{2}, \hat{x}\right) \circ \Pi_{g}^{u}\left(\hat{p}_{2}, \hat{w}_{2}\right) \circ \Pi_{g}^{s}\left(\hat{z}_{2}, \hat{p}_{2}\right) \circ \Pi_{g}^{u}\left(\hat{x}, \hat{z}_{2}\right)
$$

have no common fixed point. Thus, for $y \in V_{x}$ we have that either for $i=1$ or 2 that

$$
\Pi_{g}^{s}\left(\hat{w}_{i}, \hat{x}\right) \circ \Pi_{g}^{u}\left(\hat{p}_{i}, \hat{w}_{i}\right) \circ \Pi_{g}^{s}\left(\hat{z}_{i}, \hat{p}_{i}\right) \circ \Pi_{g}^{u}\left(\hat{x}, \hat{z}_{i}\right)(y) \neq y .
$$

This completes the proof. 
We need the following elementary result. For completeness, we give a proof in the appendix. It says roughly that two nondecreasing functions of the interval with arbitrarily small translations have no fixed points in common (this is very simple when the maps are $C^{1}$ by transversality).

Proposition 3.6. Let $\ell_{1}:[-a, a] \rightarrow \mathbb{R}$ and $\ell_{2}:[-b, b] \rightarrow \mathbb{R}$ be two non-decreasing functions and let $\phi:[-b, b] \rightarrow[-a, a]$ be also a non-decreasing function. Then for any $\varepsilon>0$ there exist $s, t,|s|,|t| \leq \varepsilon$, such that:

$$
\phi\left(\left\{x \in[-b, b]: \ell_{2}(x)+t=x\right\}\right) \cap\left\{x \in[-a, a]: \ell_{1}(x)+s=x\right\}=\emptyset .
$$

Proof. See Appendix A.

We now present the last lemma of this section and it will play a key role in the proof of our main results (Theorems $4 \mathrm{~A}$ and $4 \mathrm{~B}$ ).

Lemma 3.7. Let $\mathcal{E}$ be either $\mathcal{E}_{\omega}^{r}$ or $\mathcal{E}_{s p \text {, } \omega}^{r}$ (i.e. as in Theorems $4 A$ and $4 B$ ). Consider $f \in \mathcal{E}$ and let $\mathscr{F}_{1}^{c}$ be a periodic compact center leaf. Let $x \in \mathscr{F}_{1}^{c}$ and assume

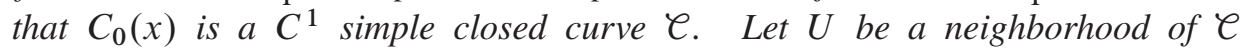
homeomorphic to an annulus and assume that a family $\Gamma$ of disjoint essential simple closed curves contained in $U$ is given, with $\ell \in \Gamma$. Then, there exist a neighborhood $V$ of $\mathcal{C}$ homeomorphic to an annulus such that for any $\varepsilon>0$ there exist $g \in \mathbb{E}$ such that

(1) $\operatorname{dist}_{C} r(f, g)<\varepsilon$,

(2) $\operatorname{supp}(f \neq g)$ is disjoint from the $f$-orbit of $\mathcal{F}_{1}^{c}$, and

(3) no curve of $\Gamma$ contained in $V$ is the accessibility class of a point in $V$, i.e. for any $y \in V, C_{0}(y, g) \neq \gamma$ for any $\gamma \in \Gamma$.

Proof. Let $x$ be as in statement of the lemma and let $x, w_{1}, w_{2}, p_{1}, p_{2}, z_{1}, z_{2}$ and $U_{1}, U_{2}$ be as in Lemma 3.3. Again, as before, let's write $\hat{w}_{i}=\mathscr{F}^{c}\left(w_{i}, f\right)$, $\hat{z}_{i}=\mathscr{F}^{c}\left(z_{i}, f\right)$, and $\hat{p}_{i}=\mathscr{F}^{c}\left(p_{i}, f\right)$. Let $V_{i}$ be the connected component of $U_{i} \cap \hat{w}_{i}$ that contains $w_{i}$ and let $V_{i}^{\prime}$ be as in Lemma 3.1. Let $W \subset \Pi_{f}^{s}\left(\hat{w}_{i}, \hat{x}\right)\left(V_{i}^{\prime}\right)$ be open and containing $x, i=1,2$, and let $\mathcal{C}_{w}=W \cap \mathcal{C}$ (we may assume that $\mathcal{C}_{w}$ is an arc). Let $\ell_{i}=\Pi_{f}^{s}\left(\hat{x}, \hat{w}_{i}\right)\left(e_{w}\right), i=1,2$ (see Figure 6 ).

In the local canonical coordinates in $V_{i}$, let $S_{i}$ be straight segments transversal to $\ell_{i}$ at $w_{i}$, and let $I_{i}=\Pi_{f}^{s}\left(\hat{w}_{i}, \hat{x}\right)\left(S_{i} \cap V_{i}^{\prime}\right)$. These arcs are transversal to $C$ at $x$. We take $V$ a compact neighborhood of $\mathcal{C}$ homeomorphic to a closed annulus, such that both $I_{i}$ crosses $V$ and intersects $C$ in just one point. We may suppose that if $\gamma \in \Gamma, \gamma \cap V \neq \emptyset$ then $\gamma \subset V$. Moreover, we redefine $I_{i}$ to be the connected component of $I_{i} \cap V$ that contains $x$ and let $S_{i}^{\prime}=\Pi_{f}^{s}\left(\hat{x}, \hat{w}_{i}\right)\left(I_{i}\right) \subset S_{i} \cap V_{i}^{\prime}$.

Let $\hat{J}_{i}=\Pi_{f}^{u}\left(\hat{z}_{i}, \hat{x}\right) \circ \Pi_{f}^{s}\left(\hat{p}_{i}, \hat{z}_{i}\right) \circ \Pi_{f}^{u}\left(\hat{w}_{i}, \hat{p}_{i}\right)\left(S_{i}\right)$ and we may also assume that $\hat{J}_{i}$ crosses $V$. Notice that $\hat{J}_{i}$ are transversal to $\ell$. Let $J_{i}$ be a connected component of $\hat{J}_{i} \cap V$ that crosses $V$ (and we may assume that $J_{i}$ intersects $\mathscr{C}$ in just 
one point). We will define functions $P_{i}: I_{i} \rightarrow J_{i}, \varphi: I_{1} \rightarrow I_{2}$, and $\psi: J_{1} \rightarrow J_{2}$ as follows. We will just define $P_{1}: I_{1} \rightarrow J_{1}$, the others are completely similar.

We order the $\operatorname{arcs} I_{1}, I_{2}, J_{1}, J_{2}$ so that all of them crosses $\mathcal{C}$ in "positive" direction.

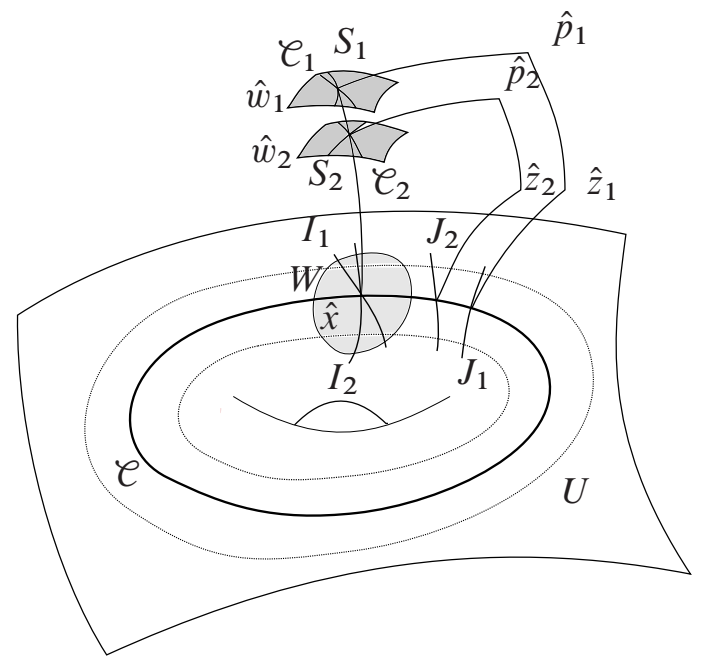

Figure 6. Perturbing closed 1-dimensional accessibility classes.

Let $\gamma \subset V$ be a curve in the family $\Gamma$. Let $x_{\gamma}$ be the closest point of $\gamma \cap J_{1}$ (in the order of $J_{1}$ ) to $J_{1} \cap \mathcal{C}$, and let $y_{\gamma}$ be the closest point of $I_{1} \cap \gamma$ (in the order of $I_{1}$ to $x=I_{1} \cap \gamma$. See Figure 7 .

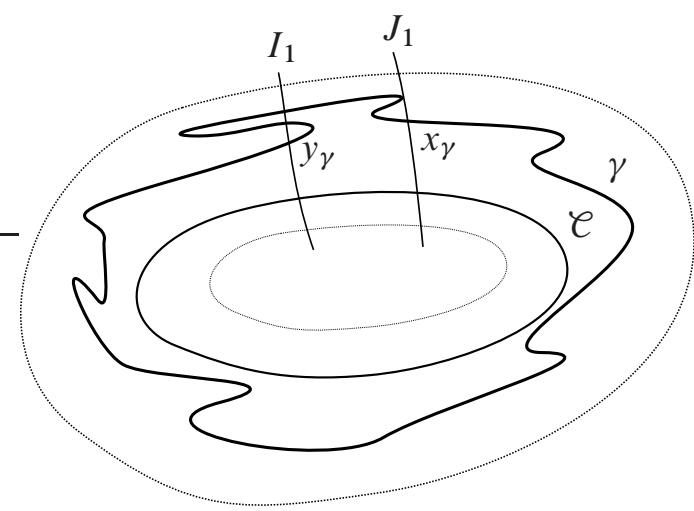

Figure 7. The map $P_{1}$.

Define $P_{1}\left(y_{\gamma}\right):=x_{\gamma}$. This is a function from

$$
\left\{y_{\gamma}: \gamma \in \Gamma\right\} \subset I_{1} \text { to }\left\{x_{\gamma}: \gamma \in \Gamma\right\} \subset J_{1} .
$$


This function is non-decreasing since for $\gamma, \eta \in \Gamma, \gamma \neq \eta$, if $y_{\gamma}<_{I_{1}} y_{\eta}$ then $x_{\gamma}<_{J_{1}} x_{\eta}$ (since each curve of $\Gamma$ separates $V$ in exactly two components and the curves in $\Gamma$ are disjoint).

Since the function is non-decreasing it may be extended to a non-decreasing function $P_{1}: I_{1} \rightarrow J_{1}$. In the same way we define $P_{2}: I_{2} \rightarrow J_{2}, \varphi: I_{1} \rightarrow I_{2}$, and $\psi: J_{1} \rightarrow J_{2}$.

Notice that for $\gamma \in \Gamma$ and $x_{\gamma}$ as before we have

$$
\psi \circ P_{1}\left(y_{\gamma}\right)=P_{2} \circ \varphi\left(y_{\gamma}\right) .
$$

Let $l_{i}: S_{i}^{\prime} \rightarrow S_{i}$ defined by

$$
l_{i}=\Pi_{f}^{u}\left(\hat{p}_{i}, \hat{w}_{i}\right) \circ \Pi_{f}^{s}\left(\hat{z}_{i}, \hat{p}_{i}\right) \circ \Pi_{f}^{u}\left(\hat{x}, \hat{z}_{i}\right) \circ P_{i} \circ \Pi_{f}^{s}\left(\hat{w}_{i}, \hat{x}\right) \mid S_{i}^{\prime} .
$$

We claim that $l_{i}$ is non-decreasing. Indeed, it is equivalent to prove that

$$
\tilde{l}_{i}=\Pi_{f}^{s}\left(\hat{w}_{i}, \hat{x}\right) \circ \Pi_{f}^{u}\left(\hat{p}_{i}, \hat{w}_{i}\right) \circ \Pi_{f}^{s}\left(\hat{z}_{i}, \hat{p}_{i}\right) \circ \Pi_{f}^{u}\left(\hat{x}, \hat{z}_{i}\right) \circ P_{i}
$$

is non-decreasing, which is equivalent to show that

$$
\hat{l}_{i}=\Pi_{f}^{s}\left(\hat{w}_{i}, \hat{x}\right) \circ \Pi_{f}^{u}\left(\hat{p}_{i}, \hat{w}_{i}\right) \circ \Pi_{f}^{s}\left(\hat{z}_{i}, \hat{p}_{i}\right) \circ \Pi_{f}^{u}\left(\hat{x}, \hat{z}_{i}\right)
$$

from $J_{i}$ to $I_{i}$ preserves orientation (since it is a diffeomorphism). Set $x_{i}=J_{i} \cap \mathcal{C}$, we know that $\hat{l}_{i}\left(x_{i}\right)=x$. If $\hat{l}_{i}$ reverse orientation, then for $y>_{J_{i}} x_{i}$ we have that $\hat{l}_{i}(y)<_{I_{i}} x$. Since $\mathcal{C}$ is essential in $U$ we get that the accessibility class of $C_{0}(y)$ must intersect $\mathcal{C}=C_{0}(x)$ and hence $\mathcal{C}$ is not an accessibility class, a contradiction.

Now, let $\varepsilon>0$ be given and let $\delta$ be as in Lemma 3.1. For $|s|<\delta$ and $|t|<\delta$ we choose $h_{1}$ and $h_{2}$ as in Lemma 3.1 so that in $V_{1}^{\prime}$ we have $h_{1}(y)=y+v_{1}, v_{1}$ in the direction of $S_{1},\left\|v_{1}\right\|=|s|$ and in $V_{2}^{\prime}$ we have $h_{2}(y)=y+v_{2}, v_{2}$ in the direction of $S_{2},\left\|v_{2}\right\|=|t|$. So, $S_{1}$ is invariant by $h_{1}$ and $S_{2}$ is invariant by $h_{2}$, and parametrizing $S_{1}$ and $S_{2}$, these maps have the form $h_{1 / S_{1}}(y)=y+s$ and $h_{2 / S_{2}}(y)=y+t$.

Now define $g=h \circ f$ where

$$
h= \begin{cases}h_{1}(x) & \text { if } x \in U_{1}, \\ h_{2}(x) & \text { if } x \in U_{2}, \\ x & \text { otherwise. }\end{cases}
$$

Notice that $\Pi_{g}^{u}\left(\hat{p}_{i}, \hat{w}_{i}\right)=h_{i} \circ \Pi_{f}^{u}\left(\hat{p}_{i}, \hat{w}_{i}\right), \Pi_{g}^{s}\left(\hat{z}_{i}, \hat{p}_{i}\right)=\Pi_{f}^{s}\left(\hat{z}_{i}, \hat{p}_{i}\right), \Pi_{g}^{u}\left(\hat{x}, \hat{z}_{i}\right)=$ $\Pi_{f}^{u}\left(\hat{x}, \hat{z}_{i}\right)$, and $\Pi_{g}^{s}\left(\hat{w}_{i}, \hat{x}\right)=\Pi_{f}^{s}\left(\hat{w}_{i}, \hat{x}\right)$.

Now, by Proposition 3.6, we may choose $s, t$ so that if $q$ is a fixed point of

$$
h_{1} \circ l_{1}=\Pi_{g}^{u}\left(\hat{p}_{1}, \hat{w}_{1}\right) \circ \Pi_{g}^{s}\left(\hat{z}_{1}, \hat{p}_{1}\right) \circ \Pi_{g}^{u}\left(\hat{x}, \hat{z}_{1}\right) \circ P_{1} \circ \Pi_{g}^{s}\left(\hat{w}_{1}, \hat{x}\right) \mid S_{1}^{\prime}
$$

then $\left[\Pi_{f}^{s}\left(\hat{x}, \hat{w}_{1}\right) \circ \varphi \circ \Pi_{f}^{s}\left(\hat{w}_{2}, \hat{x}\right)\right]^{-1}(q)$ does not contain any fixed point of $h_{2} \circ l_{2}$. 
Thus, by conjugacy with $\Pi_{g}^{s}\left(\hat{x}, \hat{w}_{1}\right)$ we have that if $q$ is a fixed point of

$$
\hat{l}_{1}:=\Pi_{g}^{s}\left(\hat{w}_{1}, \hat{x}\right) \circ \Pi_{g}^{u}\left(\hat{p}_{1}, \hat{w}_{1}\right) \circ \Pi_{g}^{s}\left(\hat{z}_{1}, \hat{p}_{1}\right) \circ \Pi_{g}^{u}\left(\hat{x}, \hat{z}_{1}\right) \circ P_{1}
$$

then $\varphi^{-1}(q)$ does not contain any fixed point of

$$
\hat{l}_{2}:=\Pi_{g}^{s}\left(\hat{w}_{2}, \hat{x}\right) \circ \Pi_{g}^{u}\left(\hat{p}_{2}, \hat{w}_{2}\right) \circ \Pi_{g}^{s}\left(\hat{z}_{2}, \hat{p}_{2}\right) \circ \Pi_{g}^{u}\left(\hat{x}, \hat{z}_{2}\right) \circ P_{2} .
$$

Now, let $\gamma \in \Gamma, \gamma \subset V$ and let $y_{\gamma}, x_{\gamma}$ as before. Then $\hat{l}_{1}\left(y_{\gamma}\right) \in C_{0}\left(x_{\gamma}, g\right)=$ $C_{0}\left(P_{1}\left(y_{\gamma}\right), g\right)$. So, if $y_{\gamma}$ is not fixed by $\hat{l}_{1}$, we have two possibilities: either

(1) $\hat{l}_{1}\left(y_{\gamma}\right)<_{1} y_{\gamma}$, or

(2) $\hat{l}_{1}\left(y_{\gamma}\right)>_{I_{1}} y_{\gamma}$.

In case (1), $\hat{l}_{1}\left(y_{\gamma}\right)$ cannot belong to $\gamma$ by the definition of $y_{\gamma}$ and so $C_{0}\left(x_{\gamma}, g\right)$ is not contained in $\gamma$.

In case (2), we conclude that the point

$$
z:=\Pi_{g}^{u}\left(\hat{z}_{1}, \hat{x}\right) \circ \Pi_{g}^{s}\left(\hat{p}_{1}, \hat{z}_{1}\right) \circ \Pi_{g}^{u}\left(\hat{w}_{1}, \hat{p}_{1}\right) \circ \Pi_{g}^{s}\left(\hat{x}, \hat{w}_{1}\right)\left(y_{\gamma}\right)
$$

satisfies $z<J_{1} x_{\gamma}$ and so does not belong to $\gamma$ which implies that $C_{0}\left(y_{\gamma}, g\right)$ is not contained in $\gamma$.

Finally, assume that $y_{\gamma}$ is fixed by $\hat{l}_{1}$ and let $\bar{x}_{\gamma}$ be the closest point of $\gamma \cap I_{2}$ (in the order of $I_{2}$ ) to $x$. Then we know that $\bar{y}_{\gamma}$ is not fixed by $\hat{l}_{2}$ and we apply the previous argument. Thus, no curve $\gamma \in \Gamma$ is an accessibility class. The proof is finished.

\section{Proof of Theorem 2}

Let $r \geq 2$ and let $\mathcal{E}$ be as in Theorem 2, i.e. $\mathcal{E}$ is $\mathcal{E}^{r}, \mathcal{E}_{m}^{r}, \mathcal{E}_{\omega}^{r}, \mathcal{E}_{s p}^{r}$ or $\mathcal{E}_{s p, \omega}^{r}$. We have to prove the set $\mathcal{R}_{0}$ of diffeomorphisms in $\mathcal{E}$ having no trivial accessibility classes is $C^{1}$ open and $C^{r}$ dense. This result is a consequence of Lemma 3.5, as follows.

Let's consider $\Gamma_{0}: \mathscr{E} \rightarrow \mathcal{C}(M)=\{$ compact subsets of $M$ \} (endowed with the Hausdorff topology)

$$
\Gamma_{0}(f)=\{x \in M: A C(x) \text { is trivial }\} .
$$

We observe that $\Gamma_{0}(f)$ is indeed a compact set, it follows from Corollary 2.11.

Lemma 4.1. The map $\Gamma_{0}$ is upper semicontinuous, i.e. given $f \in \mathcal{E}$ and a compact set $K$ such that $\Gamma_{0}(f) \cap K=\emptyset$ then there exists a neighborhood $U(f)$ of $f$ in $\varepsilon$ (which is also $C^{1}$ open) such that $\Gamma_{0}(g) \cap K=\emptyset$ for all $g \in \mathcal{U}(f)$. 
Proof. Let $y \notin \Gamma_{0}(f)$. From Corollary 2.13 there exist $U(y)$ and $U_{y}(f)$ (which is also $C^{1}$ open) such that for any $g \in U_{y}(f)$ and $z \in U(y)$ we have that $A C(z, g)$ is non-trivial.

Now, consider the family of $U_{y}$ with $y \in K$. We may cover $K$ with finitely many of them, say $K \subset \bigcup_{i=1}^{n} U_{y_{i}}$.

Let $U(f) \subset \bigcap_{i=1}^{n} \mathcal{U}_{y_{i}}(f)$. If $g \in \mathcal{U}(f)$ and $z \in K$ then $z \in U_{y_{i}}$ and $g \in U_{y_{i}}(f)$ for some $i$ and so $A C(z, g)$ is non-trivial. The proof of the lemma is complete.

By taking $K=M$ in the previous lemma, we get:

Corollary 4.2. If for some $f \in \mathcal{E}$ we have that $\Gamma_{0}(f)=\emptyset$ then there is a neighborhood $U(f) \subset \mathcal{E}$ (which is $C^{1}$ open) such that for any $g \in \mathcal{U}(f)$ we have $\Gamma_{0}(g)=\emptyset$.

Now, we are ready to conclude:

Proof of Theorem 2. Let $\mathscr{I}_{0}$ be the set of continuity points of $\Gamma_{0}$. This is a residual set in $\mathscr{E}$ (since $\mathcal{E}$ with the $C^{r}$ topology is a Baire space). We claim that if $f \in \mathscr{E}_{0}$ then $\Gamma_{0}(f)=\emptyset$. Otherwise, let $x \in \Gamma_{0}(f)$ and we may assume that $x$ belongs to a periodic compact center leaf (see Lemma 2.4).

Indeed, by the continuity of $\Gamma_{0}$ at $f$ we have that for any neighborhood $V$ of $x$ there exists $U(f)$ such that for any $g \in \mathcal{U}(f)$ there is $x_{g} \in V$ such that $A C\left(x_{g}, g\right)$ is trivial. A direct application of Lemma 3.5 yields a contradiction and the claim is proved.

From this and Corollary 4.2 we get that the set

$$
\mathcal{R}_{0}=\left\{f \in \mathcal{E}: \Gamma_{0}(f)=\emptyset\right\}
$$

is $C^{1}$ open and $C^{r}$ dense in $\mathcal{E}$. This set $\mathcal{R}_{0}$ is just the set of diffeomorphisms where any accessibility class is nontrivial.

\section{The accessibility class of periodic points}

Through this section, we consider $\mathcal{E}$ to be either $\mathcal{E}^{r}, \mathcal{E}_{m}^{r}, \mathcal{E}_{\omega}^{r}, \mathcal{E}_{s p}^{r}$ or $\mathcal{E}_{s p, \omega}^{r}$ and with $\operatorname{dim} E^{c}=2$.

Let $f \in \mathcal{E}$ and let $\mathcal{F}_{1}^{c}$ be a compact periodic center leaf of period $k$ and let $p \in \mathscr{F}_{1}^{c}$ be a periodic point of $f$. Let $U$ be a neighborhood of $p$ in $M$. We denote by $C_{0}(p, U, f)$ the local accessibility class of $p$, that is, the set $y \in \mathscr{F}_{1}^{c}$ that can be joined to $p$ by $s u$-path contained in the neighborhood $U$ of $p$.

We will classify the periodic points with respect to its behaviour on the central leaf. In particular we say

- $p$ is center-hyperbolic of saddle type if $p$ is hyperbolic of saddle type with respect to $f_{/ \mathscr{F}_{1}^{c}}^{k}$, 
- $p$ is center-attractor or center-repeller if $p$ is attractor or repeller w.r.t. $f_{/ F_{1} c}^{k}$,

- $p$ is center-elliptic if $p$ is elliptic w.r.t. $f_{/ \mathscr{F}_{1}^{c}}^{k}$.

Assume that $p$ is a center-hyperbolic periodic point of saddle type $f_{/ \mathscr{F}_{1}^{c}}^{k}$. We denote by $C W^{s}(p)$ the stable manifold of $p$ with respect to $f_{\mid \mathcal{F}_{1}^{c}}^{k}$. We write $C E_{p}^{s} \subset T_{p} \mathcal{F}_{1}^{c}$ the tangent space to $C W^{s}(p)$. Analogously, we denote $C W^{u}(p)$ the unstable manifold of $p$ with respect to $f_{/ \mathcal{F}_{1}^{c}}^{k}$ and $C E_{p}^{u} \subset T_{p} \mathcal{F}_{1}^{c}$ the tangent space to $C W^{u}(p)$.

We say that a periodic point $p$ of period $\tau(p)$ of $f$ is generic if:

- $p$ is hyperbolic in the case $\mathcal{E}=\mathcal{E}^{r}$ or $\mathcal{E}_{s p}^{r}$.

- -1 and 1 are not eigenvalues of $D f_{p}^{\tau(p)}$ in the case $\mathcal{E}=\mathcal{E}_{m}^{r}, \mathcal{E}_{\omega}^{r}$ or $\mathcal{E}_{s p, \omega}^{r}$.

Lemma 5.1. There exists a residual set $\mathscr{E}_{1}$ in $\mathcal{E}$ such that if $f \in \mathscr{E}_{1}$ and $p$ is a centerhyperbolic periodic point of saddle type of $f$ for $f_{\mid \mathcal{F}_{1}^{c}}^{k}$ then there exist neighborhoods $U_{c}$ and $U$ of $p, U_{c}$ in $\mathscr{F}^{c}(p)$ and $U$ in $M$, such that $U_{c} \backslash\left(C W_{\mathrm{loc}}^{s}(p) \cup C W_{\mathrm{loc}}^{u}(p)\right)$ has four connected component, $U \cap \mathscr{F}^{c}(p) \subset U_{c}$, and $C_{0}(p, U, f)$ is not contained in $C W_{\mathrm{loc}}^{s}(p) \cup C W_{\mathrm{loc}}^{u}(p)$.

We say that a periodic point $p$ as in the previous lemma satisfies the Property $(L)$.

Proof. Let $H_{n}=\left\{f \in \mathcal{E}\right.$ : all points in $\operatorname{Fix}\left(f^{n}\right)$ are generic and every centerhyperbolic periodic point $p \in \operatorname{Fix}\left(f^{n}\right)$ of saddle type satisfies Property $\left.(L)\right\}$.

Claim. $H_{n}$ is open and dense in $\mathcal{E}$. In fact, notice that $H_{n}^{0}=\{f \in \mathcal{E}$ : Fix $\left(f^{n}\right)$ generic $\}$ is open and dense in $\mathcal{E}$. Thus, to prove the claim it is enough to show that $H_{n}$ is open and dense in $H_{n}^{0}$. It is immediate that $H_{n}$ is open in $H_{n}^{0}$. Let us show that $H_{n}$ is dense. Let $f \in H_{n}^{0}$. We know that there are finitely many center-hyperbolic periodic points in $\operatorname{Fix}\left(f^{n}\right)$. Choose a neighborhood $U_{c}$ for each one as in Property $(L)$. By similar arguments as Lemma 3.5 it is not difficult to get $g \in H_{n}$ arbitrarily close to $f$ satisfying Property $(L)$.

Finally, set $\mathscr{P}_{1}=\cap_{n \geq 0} H_{n}$ and the lemma is proved.

Theorem 5.2. There exists a residual subset $\mathcal{R}_{*}$ in $\mathcal{E}$ such that if $f \in \mathcal{R}_{*}$ and $p$ is a periodic point which is neither a center-attractor nor a center-repeller for $f_{/ \mathscr{F}_{1}^{c}}^{k}$ on a compact periodic center leaf $\mathcal{F}_{1}^{c}$ then $C_{0}(p, f)$ is open. Moreover, if $p$ is center-hyperbolic of saddle type then there exist an open set $V$ in $M$ (contained in a ball around $p$ ) and a neighborhood $U(f)$ (which is also $C^{1}$ open) such that for any $g \in \mathcal{U}(f)$, we have $V \subset A C\left(p_{g}, g\right)$ where $p_{g}$ is the continuation of $p$ for $g \in U(f)$.

Proof. Let $\mathcal{R}_{*}=\mathcal{R}_{0} \cap \mathcal{E}_{1}$ where $\mathcal{R}_{0}$ is as in Theorem 2 (see also (4.2)). Let $f \in \mathcal{R}_{*}$ and let $p$ be a periodic point of $f$. Since $f \in \mathcal{R}_{*}, C_{0}(p):=C_{0}(p, f)$ is either open or a one dimensional $C^{1}$ submanifold. 
Assume first that $p$ is a center-elliptic periodic point of $f_{/ \mathscr{F}_{1}^{c}}^{k}$ of period $\tau(p)$. Now, since $C_{0}(p)$ is invariant under $f^{\tau(p)}$ and there is no invariant direction of $D f_{/ \mathscr{F}^{c}(p)}^{\tau(p)}$ we easily conclude that $C_{0}(p)$ is open.

Assume now that $p$ is a center-hyperbolic periodic point of $f_{/ \mathscr{F}_{1}^{c}}^{k}$ of saddle type of period $\tau(p)$ and assume, by contradiction, that $C_{0}(p)$ is not open, that is, $C_{0}(p)$ is a one dimensional $C^{1}$ submanifold. Then $p$ satisfies Property $(L)$. This implies that there exists a non trivial connected set $C \subset C_{0}(p, U, f) \subset C_{0}(p)$ and not contained in $C W_{\mathrm{loc}}^{s}(p) \cup C W_{\mathrm{loc}}^{u}(p)$.

On the other hand, $T_{p} C_{0}(p)$ must be an invariant direction (by the invariance of $\left.C_{0}(p)\right)$ by $D f_{p}^{\tau(p)}$ and so $T_{p} C_{0}(p)=C E_{p}^{s}$ or $C E_{p}^{u}$. Assume that $T_{p} C_{0}(p)=C E_{p}^{s}$. Thus, $C_{0}(p)$ is locally a graph around $p$ (via the exponential map) of a map from $C E_{p}^{s} \rightarrow C E_{p}^{u}$.

Now, this graph is not contained in $C W_{\mathrm{loc}}^{s}(p)$ (since $p$ satisfies Property $(L)$ and the connected set $\left.C \subset C_{0}(p, U, f)\right)$. But notice that this graph is locally invariant, by the invariance of $C_{0}(p)$. This is a contradiction since there is a unique locally invariant graph, namely $C W_{\mathrm{loc}}^{s}(p)$. Analogously, if $T_{p} C_{0}(p)=C E_{p}^{u}$ we use the same argument for $f^{-1}$. Thus, we have proved that $C_{0}(p)$ is open.

As the accessibility class $C_{0}(p)$ of a center-hyperbolic periodic point of saddle type $p$ is open and that $C W_{\text {loc }}^{s}(p)$ and $C W_{\text {loc }}^{u}(p)$ intersect $C_{0}(p)$ then, by invariance we get that $C W^{s}(p)$ and $C W^{u}(p)$ are contained in $C_{0}(p)$.

From Property $(L)$, we know that there exists $y_{f} \in U_{c} \backslash C W_{\mathrm{loc}}^{s}(p) \cup C W_{\mathrm{loc}}^{u}(p)$ so that $y_{f} \in C_{0}(p, U, f)$. Let's order the four connected component clockwise beginning with the one that contains $y_{f}$. See Figure 8 .

Let $B_{c}\left(y_{f}\right)$ be a ball centered in $y_{f}$ contained in $U_{c} \backslash\left(C W_{\mathrm{loc}}^{s}(p) \cup C W_{\mathrm{loc}}^{u}(p)\right)$, that is, $B_{c}\left(y_{f}\right) \subset(I)$.

We know (see Lemma 2.7) that there exists a continuous map $\gamma: B_{c}(p) \times[0,1] \rightarrow$ $U_{c}$ such that

- $\gamma(z, 0)=z$,

- $\gamma(z, 1) \subset B_{c}\left(y_{f}\right)$, and

- $\gamma(z, t) \subset C_{0}(z, U, f)$.

Let $V_{c}$ be an open set such that $\overline{V_{c}} \subset B_{c}(p) \cap$ (III). Thus, for any $z \in V_{c}$ we have for some $t_{0}$ that $\gamma\left(z, t_{0}\right) \in\left(C W_{\mathrm{loc}}^{s}(p) \cup C W_{\mathrm{loc}}^{u}(p)\right) \subset C_{0}(p)$ and so $V_{c} \subset C_{0}(p)$.

Finally, we saturate $V_{c}$ by local (strong) stable and unstable manifolds to obtain an open set $V \subset M$. This set $V$ satisfies the requirement of the theorem for $U(f)$ small enough by the continuation of center leaves, strong stable and unstable leaves, the continuation of $p$, and the continuation of $\gamma$ (see also Lemma 2.12) and so Property $(L)$ is open. The theorem is proved. 


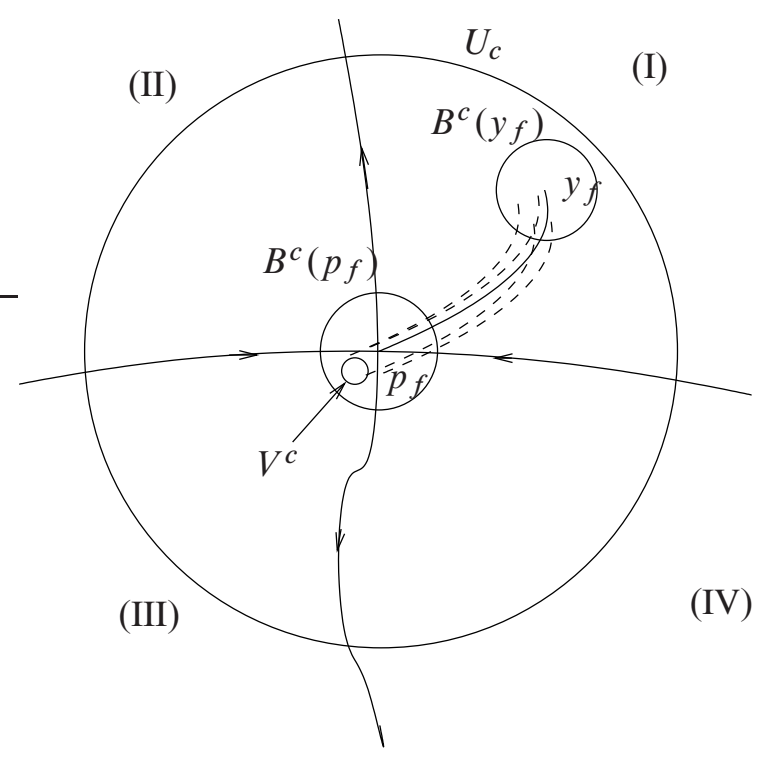

Figure 8. Accessibility class of hyperbolic periodic points.

Let $f \in \mathcal{R}_{*}$ and let $p$ be a center-hyperbolic periodic point of saddle type (belonging to a periodic compact center leaf). Let $U(f)$ corresponding to $p$ and $f$ in Theorem 5.2 (we denote by $p_{g}$ the continuation of $p$ for $g \in U(f)$ ).

Let $\Gamma_{1}: U(f) \rightarrow \mathcal{C}(M)$,

$$
\Gamma_{1}(g)=M \backslash A C\left(p_{g}, g\right) .
$$

Notice that $\Gamma_{1}$ is well defined since $A C\left(p_{g}, g\right)$ is open for $g \in \mathcal{U}(f)$.

Proposition 5.3. The map $\Gamma_{1}$ is upper semicontinuous, i.e. given $g \in U(f)$ and a compact set $K$ such that $\Gamma_{1}(g) \cap K=\emptyset$ then there exists a neighborhood $\mathcal{V}(g) \subset \mathcal{U}(f)$ (which is also $C^{1}$ open) such that for any $h \in \mathcal{V}(g)$ we have that $\Gamma_{1}(h) \cap K=\emptyset$.

Proof. Let $V$ be the fixed open set in $M$ from Theorem 5.2, that is, $V \subset A C\left(p_{g}, g\right)$ for every $g \in U(f)$. Let $g \in U(f)$ and $K$ compact with $\Gamma_{1}(g) \cap K=\emptyset$ be given. Let $y \in K$, then there exists $U_{y}$ and $U_{y}(g)$ such that for any $h \in U_{y}(g)$ and any $z \in U_{y}$ we have that $A C(z, h) \cap V \neq \emptyset$ (see Lemma 2.12), in other words $U_{y} \subset A C\left(p_{h}, h\right)$ for any $h \in U_{y}(g)$.

Now, cover $K$ with finitely many of these open sets $U_{y}$, that is, $K \subset \bigcup_{i=1}^{n} U_{y_{i}}$. Let $\mathcal{V}(g)=\bigcap_{i=1}^{n} \mathcal{U}_{y_{i}}(g)$. Then, for every $h \in \mathcal{V}(g)$ we have that $K \subset A C\left(p_{h}, h\right)$. The proof of proposition in finished. 
Corollary 5.4. Assume that for $g \in \mathcal{U}(f)$ we have that $\Gamma_{1}(g)=\emptyset$, then $g$ is accessible. Moreover, there exists $\mathcal{V}(g)$ (which is also $C^{1}$ open) such that any $h \in \mathcal{V}(g)$ is accessible.

\section{Proof of Theorems $3 A$ and $3 B$}

For simplicity, we prove both theorems together since the proof is essentially the same. Let $\mathcal{E}$ be either $\mathcal{E}_{A}^{r}$ or $\mathcal{E}_{A, m}^{r}$ and with $\operatorname{dim} E^{c}=2$, that is, the set of $\mathcal{E}^{r}$ or $\mathcal{E}_{m}^{r}$ where $\operatorname{dim} E^{c}=2$ and having a compact periodic leaf so that the dynamics in this leaf (in the period) is both Axiom A and sinkless. Let $\mathcal{R}_{0}$ be as in Theorem 2 and $\mathcal{R}_{*}$ as in Theorem 5.2 (both restricted to $\mathcal{E}_{A}^{r}$ or $\mathcal{E}_{A, m}^{r}$ ). Thus, $\mathcal{R}_{0} \cap \mathcal{R}_{*}$ is residual in $\mathcal{E}$. Let $f \in \mathcal{R}_{0} \cap \mathcal{R}_{*}$ (although in the proof of Theorem 5.2 we construct $\mathcal{R}_{*} \subset \mathcal{R}_{0}$ ). We will prove that $f$ is accessible. Let's see the properties we know for $f$ :

- Any accessibility class is nontrivial.

- The accessibility classes of center-hyperbolic periodic points of saddle type of $f$ are open.

- If $p$ is a center-hyperbolic periodic point of saddle type, then it satisfies Property $(L)$.

- There exists a compact periodic center leaf $\mathscr{F}_{1}^{c}$ such that $f_{/ \mathscr{F}_{1}^{c}}^{k}$ is an Axiom-A diffeomorphism and sinkless, where $k$ is the period of $\mathscr{F}_{1}^{c}$.

- If $p$ is center-hyperbolic periodic point of saddle type, then the stable and unstable manifolds in the center leaf $C W^{s}(p)$ and $C W^{u}(p)$ are contained in the accessibility class of $p$.

Let's denote by $\Lambda$ a basic piece from Smale's spectral decomposition of $f_{\mid F_{1} c}^{k}$ which is not a periodic center-repeller. Recall that the stable and unstable manifolds $C W^{s}(\mathcal{O}(p))$ and $C W^{u}(\mathcal{O}(p))$ are dense in $\Lambda$ for any periodic point $p \in \Lambda$. Let $x \in \Lambda$ be any point. We know that $C_{0}(x)$ is open or a one dimensional $C^{1}$ manifold without boundary containing $x$. In any case, we have that it intersects $C W^{s}(\mathcal{O}(p))$ or $C W^{u}(\mathcal{O}(p))$ and therefore $C_{0}(x) \cap C_{0}\left(f^{i}(p)\right) \neq \varnothing$ for some $i$. Therefore, $C_{0}(x)=C_{0}\left(f^{i}(p)\right)$. This means that $\Lambda$ is contained in (the open set) $\cup_{q \in \mathcal{O}(p)} C_{0}(q)$ for $p \in \Lambda$ periodic. By the invariance of $\cup_{q \in \mathcal{O}(p)} C_{0}(q)$ we also have that $C W^{s}(\Lambda)$ and $C W^{u}(\Lambda)$ are contained in $\cup_{q \in \mathcal{O}(p)} C_{0}(q)$. Let $F_{0}$ be the set of periodic centerrepellers which is a finite set. Let $F_{1}$ be the set of center-hyperbolic periodic points of saddle type. Since there are no periodic center-attractor we have that $\operatorname{Per}\left(f_{/ \mathcal{F}_{1}^{c}}\right)=F_{0} \cup F_{1}$.

Since every point in $\widetilde{F}_{1}^{c}$ is contained in the stable manifold (inside the center leaf) of the basic pieces, we conclude that $\widetilde{F}_{1}^{c} \backslash F_{0} \subset \cup_{p \in F_{1}} C_{0}(p)$ and that $C_{0}(p)$ is open for any periodic point in $F_{1}$. By connectedness we conclude that $\mathscr{F}_{1}^{c} \backslash F_{0} \subset C_{0}(p)$ (for any periodic point $p \in F_{1}$ ). Since the accessibility classes are non trivial for 
every $q \in F_{0}$, we have that $C_{0}(q) \cap C_{0}(p) \neq \emptyset$ and so $C_{0}(q)=C_{0}(p)$. Thus $\mathscr{F}_{1}^{c}=C_{0}(p)$ for any $p \in F_{1}$. That is, the center leaf $\mathscr{F}_{1}^{c}$ is just one center accessibility class and by Lemma 2.4 we have that $f$ is accessible, as we claimed.

Finally, since $f \in \mathcal{R}_{*}$ and fixing a center-hyperbolic periodic point of saddle type $p$ of $f$, in the setting of Section 5, we have a neighborhood $U(f)$ and a map $\Gamma_{1}$ defined in $U(f)$ as in (5.1). Due to what we just proved $\Gamma_{1}(f)=\emptyset$ holds and so by Corollary 5.4 there exists $\mathcal{V}(f)$ such that any $g \in \mathcal{V}(f)$ is accessible. Thus,

$$
\widehat{\mathcal{R}}=\bigcup_{f \in \mathcal{R}_{0} \cap \mathcal{R}_{*}} \mathcal{V}(f)
$$

is $C^{1}$ open and $C^{r}$ dense in $\mathcal{E}$ and formed by accessible diffeomorphisms. This completes the proof of Theorems 3A and 3B.

6.1. Examples. We present here some examples where Theorems $3 \mathrm{~A}$ and $3 \mathrm{~B}$ apply.

Example 6.1. This example can be thought as a conservative version of the well known Shub's example on $\mathbb{T}^{4}$ [34].

Consider the Lewowicz family (see [22]) of conservative diffeomorphisms on $\mathbb{T}^{2}$ :

$$
f_{c}(x, y)=\left(2 x-\frac{c}{2 \pi} \sin (2 \pi x)+y, x-\frac{c}{2 \pi} \sin (2 \pi x)+y\right), \quad c \in \mathbb{R} .
$$

Notice that when $c=0 f_{c}$ is Anosov and when $1<c<5$ the fixed point at $(0,0)$ is an elliptic fixed point. We just consider for instance $c \in[0,2]$. From this family it is not difficult to construct a continuous map

$$
g: \mathbb{T}^{2} \rightarrow \operatorname{Diff}^{\infty}\left(\mathbb{T}^{2}\right)
$$

such that for two points $p, q \in \mathbb{T}^{2}$ given, we have $g(p)=f_{0}$ and $g(q)=f_{2}$. Now, given $r \geq 2$, consider a conservative Anosov diffeomorphism $A: \mathbb{T}^{2} \rightarrow \mathbb{T}^{2}$ having $p, q \in \mathbb{T}^{2}$ as fixed points and with enough strong expansion and contraction so the map

$$
F: \mathbb{T}^{2} \times \mathbb{T}^{2} \rightarrow \mathbb{T}^{2} \times \mathbb{T}^{2} \text { defined as } F(x, y)=\left(A(x), g_{x}(y)\right)
$$

belongs to $\mathcal{E}_{A, m}^{r}\left(\mathbb{T}^{2} \times \mathbb{T}^{2}\right)$. The center foliation is thus $\{x\} \times \mathbb{T}^{2}$ and at $\mathscr{F}^{c}(p)=$ $\{p\} \times \mathbb{T}^{2}$ the map $F$ supports an Anosov (and hence is an Axiom-A diffeomorphism without periodic attractors). Our theorem implies that a generic arbitrarily small $C^{r}$ perturbation (preserving the Lebesgue measure on $\mathbb{T}^{2} \times \mathbb{T}^{2}$ ) is stably ergodic. The same example can be considered also just in the skew-product setting.

Remark 6.2. Notice that due to the presence of an elliptic point on $\{q\} \times \mathbb{T}^{2}$ the center bundle $E^{c}$ does not admit any dominated splitting. By the result in [4] we may find a perturbation of $F$ (and stably ergodic) such that the center Lyapunov exponent is nonzero. This implies that the center foliation (which is two dimensional) is not absolutely continuous. 
Theorem $3 \mathrm{~A}$ admits some generalizations or different versions. We just give some examples and an idea of how to prove stable ergodicity.

Example 6.3. Consider $f: \mathbb{T}^{2} \rightarrow \mathbb{T}^{2}$ a conservative Anosov diffeomorphism and let $F_{0}: \mathbb{T}^{2} \times \mathbb{S}^{1} \rightarrow \mathbb{T}^{2} \times \mathbb{S}^{1}$ as $F_{0}=f \times i d$. This is a conservative partially hyperbolic diffeomorphism on $\mathbb{T}^{3}$ with one dimensional center and the center foliation is by circles. Let $p$ be a fixed point of $f$. It is not difficult to construct a (conservative) perturbation $F$ of $F_{0}$ such that on the corresponding $\mathscr{F}^{c}(p, F)$ the dynamics is a north-south Morse-Smale dynamics and $F$ satisfies the same generic conditions as in Theorem 3A. Then, the map $F \times F: \mathbb{T}^{6} \rightarrow \mathbb{T}^{6}$ belongs to $\varepsilon_{m}^{r}\left(\mathbb{T}^{6}\right)$, the center foliation is by $\mathbb{T}^{2}$ and $F_{/ \mathcal{F} c}(p, p)$ is an Axiom-A diffeomorphism (the product of the two Morse-Smale on the circle). Theorem 3A does not apply in this case because $F_{/ \mathscr{F} c}(p, p)$ have a center-attracting and a center-repelling periodic point. Nevertheless, by the similar arguments as in the proof of Theorem $3 \mathrm{~A}$ one gets that the union of the accessibility classes of the two center-hyperbolic saddles in $\mathscr{F}^{c}(p, p)$ is open and if it is not the whole center leaf $\mathscr{F}^{c}(p, p)$ then its complement consist of a closed $C^{1}$ curve which is a connection between the attractor and the repeller. Since this curve does not separate the leaf $\mathscr{F}^{c}(p, p)$ we have that the union of the accessibility classes of the two center-hyperbolic saddles is just one accessibility class $C_{0}(q)$, for $q$ any periodic saddle, which is open and $A C(q)$ has full measure in $\mathbb{T}^{6}$. This means that $F$ is essentially accessible and hence ergodic. Since the above situation is $C^{r}$ open we conclude that $F$ is $C^{r}$ stably ergodic.

The same argument also applies to the next example.

Example 6.4. Consider $f_{0}: M \rightarrow M$ to be the time one map of the suspension of a conservative Anosov diffeomorphisms on $\mathbb{T}^{2}$. This is a conservative partially hyperbolic diffeomorphism whose leaves of the center foliation are the orbits of the suspension. Let $p$ be a fixed point of the Anosov and the center leaf $\mathscr{F}^{c}\left(p, f_{0}\right)$ is a circle where $f_{0}$ is the identity. We then find a conservative and generic perturbation $f$ of $f_{0}$ such that $f$ restricted to $\mathscr{F}^{c}(p, f)$ is a Morse-Smale system. The diffeomorphism $f \times f: M \times M \rightarrow M \times M$ belongs to $\varepsilon_{m}^{r}(M \times M)$ with two dimensional center leaves and in the leaf $\mathscr{F}^{c}((p, p), f \times f)$ is an Axiom-A on a two torus and in the same situation as the previous example. The same argument yields that $f \times f$ is stably ergodic.

\section{Proof of Theorems $4 A$ and $4 B$}

The proof of Theorems 4A and 4B are essentially the same. Indeed, the important thing we need is that if $L=f^{k}(L)$ is a periodic compact leaf then $f_{\mid L}^{k}$ preserves area. This automatically holds in the case of conservative skew products. We will see that this property also holds if a symplectic form is preserved. 
In this section we denote by $\mathcal{E}$ either $\mathcal{E}_{\omega}^{r}$ or $\mathcal{E}_{s p, \omega}^{r}$ and with $\operatorname{dim} E^{c}=2$. The key fact about preserving a symplectic form $\omega$ in $\mathcal{E}_{\omega}^{r}$ is the following folklore result (see [39, Lemma 2.5]):

Lemma 7.1. Let $f: M \rightarrow M$ be a partially hyperbolic diffeomorphism preserving a symplectic form $\omega$. Then $\omega_{/ E^{c}}$ is non degenerate (and so symplectic). In particular if $f$ is dynamically coherent and $\operatorname{dim} E^{c}=2$ then $\omega$ is an area form in $\mathscr{F}^{c}(x)$ for any $x$. Furthermore, if $\mathscr{F}^{c}(x)$ is k-periodic then $f_{/ \mathcal{F}^{c}(x)}^{k}$ is a conservative diffeomorphism.

Throughout this section in order to simplify notation we omit the word center when we refer to the classification of periodic point in a center leaf in Section 5.

The next lemma says that generically we have compact leaves with periodic points.

Lemma 7.2. There exists a $C^{1}$ open and $C^{r}$ dense set $\mathscr{F}_{2}$ in $\mathbb{E}$ such that if $f \in \mathscr{E}_{2}$ then there exists a periodic compact leaf having a hyperbolic periodic point.

Proof. Notice that the set in $\mathcal{E}$ having a hyperbolic periodic point on some compact periodic leaf is $C^{1}$ open.

Let $f_{0} \in \mathcal{E}$ and let $\mathscr{F}_{1}^{c}$ be a compact periodic leaf. For simplicity we assume it is fixed. We may assume also that $\mathscr{F}_{1}^{c}$ is orientable (otherwise we go to the double covering). If $\mathscr{F}_{1}^{c}$ is not the two torus then $f_{0 / \mathcal{F}_{1}^{c}}$ has periodic points. Let $f \in \mathcal{E}$ be a Kupka-Smale diffeomorphism and arbitrarily $C^{r}$ close to $f_{0}$. It follows that there is a hyperbolic periodic point in $\mathscr{F}_{1}^{c}(f)$ since once we have elliptic periodic points we have hyperbolic periodic points (see [40]).

If $\mathscr{F}_{1}^{c}$ is the two torus and $f_{0}$ has no periodic points in $\mathscr{F}_{1}^{c}$, then by composing with a translation in the torus (and extending this perturbation on $\mathscr{F}_{1}^{c}$ to $M$ ) we may change the mean rotation number (the mean rotation number of the composition of two maps is the sum of the mean rotation number of each one) to get a rational mean rotation number. Using a result by Franks [16] we get a periodic point, which by perturbation we may assume that it is hyperbolic or elliptic. And then we argue as before.

Remark 7.3. In this situation we are working with $\left(\mathcal{E}_{\omega}^{r}\right.$ or $\left.\mathcal{E}_{s p, \omega}^{r}\right)$, if $p$ is a hyperbolic periodic point of $f$ then for the restriction to the center manifold that contains $p$ we have that $p$ is a hyperbolic periodic point of saddle type (since the restriction to the center manifold preserves area).

Let $\mathcal{R}_{0}$ from Theorem 2 , and let $\mathcal{K} \&$ be the set of Kupka-Smale diffeomorphisms in $\mathcal{E}$ which is a residual set, and let $\mathcal{R}_{*}$ as in Theorem 5.2. We consider

$$
f \in \mathcal{R}_{0} \cap \mathcal{R}_{*} \cap \mathcal{E}_{2} \cap \mathcal{K} \delta
$$

and let $\mathscr{F}_{1}^{c}$ be a compact $k$-periodic leaf containing a hyperbolic periodic point $p$, $\mathscr{F}_{1}^{c}=\mathscr{F}^{c}(p)$. 
Let $\mathcal{U}(f)$ be as in Theorem 5.2 and let $\Gamma_{1}$ as in (5.1). Let $\mathcal{R}_{1}(f) \subset \mathcal{U}(f)$ be the residual subset of continuity points of $\Gamma_{1}$.

We will assume for simplicity that the compact leaf $S=\mathscr{F}^{c}(p, f)$ is fixed by $f$. When $g$ varies on $U(f)$ the compact leaf $\mathscr{F}^{c}\left(p_{g}, g\right)$ varies continuously (by a homeomorphism on $M$ close to the identity), and thus there is a natural identification between $\mathscr{F}^{c}\left(p_{g}, g\right)$ with $\mathscr{F}^{c}(p, f)$ as the surface $S$. In order to avoid unimportant technicalities we will assume that $\mathscr{F}^{c}\left(p_{g}, g\right)=S$ for any $g \in \mathcal{U}(f)$. Now consider the following functions $\Gamma_{2}, \Gamma_{3}: U(f) \rightarrow \mathcal{C}(S)$, where $\mathcal{C}(S)$ is the set of compact subset of $S$ with the Hausdorff topology:

$$
\Gamma_{2}(g)=S \backslash C_{0}\left(p_{g}, g\right) \quad \text { and } \quad \Gamma_{3}(g)=\overline{C_{0}\left(p_{g}, g\right)},
$$

where $C_{0}\left(p_{g}, g\right)$ is the connected component of $C\left(p_{g}, g\right)$ that contains $p_{g}$.

Lemma 7.4. The function $\Gamma_{2}$ is upper semicontinuous and the function $\Gamma_{3}$ is lower semicontinuous. That is, given $g \in \mathcal{U}(f)$, a compact set $K \subset S$ and an open set $U \subset S$ such that $K \cap \Gamma_{2}(g)=\emptyset$ and $U \cap \Gamma_{3}(g) \neq \emptyset$ then there exists $U(g)$ such that $K \cap \Gamma_{2}(h)=\emptyset$ and $U \cap \Gamma_{3}(h) \neq \emptyset$ for any $h \in \mathcal{U}(g)$.

Proof. The proof that $\Gamma_{2}$ is upper semicontinuous is similar as the proof of Proposition 5.3. Let $V^{\prime}=V \cap S$ where $V$ is as in Theorem 5.2 and let $K \subset S$ a compact set as in statement, that is $K \subset C_{0}\left(p_{g}, g\right)$. Let $y \in K$. There exists $U_{y} \subset S$ and $\mathcal{U}_{y}(g)$ such that for any $z \in U_{y}$ and $h \in \mathcal{U}_{y}(g)$ we have that $A C(z, h) \cap V^{\prime} \neq \emptyset$. On the other hand we can assume that $U_{y} \subset C_{0}\left(p_{g}, g\right)$ and this means that the $s u$ path (of $g$ ) joining $z \in U_{y}$ with $V^{\prime}$ when lifted to the covering $\widetilde{M}$ is a path that starts and ends on a same center leaf (which projects to $S$ ) and so the same happens for $h$ near $g$. This implies that $U_{y} \subset C_{0}\left(p_{h}, h\right)$ for any $h \in \mathcal{U}_{y}(g)$. Then, covering $K$ with finitely many sets $U_{y}$ and taking the corresponding intersection of the $U_{y}(g)$ we conclude the statement on $\Gamma_{2}$.

Let's prove the semicontinuity of $\Gamma_{3}$. Let $U \subset S$ be an open set such that $U \cap \Gamma_{3}(g) \neq \emptyset$. In particular $U \cap C_{0}\left(p_{g}, g\right) \neq \emptyset$. Let $y$ be in this intersection and let $U_{y}$ and $U_{y}(g)$ as before. We may assume that $U_{y} \subset U$. Then, for any $h \in \mathcal{U}_{y}(g)$ we have that $U_{y} \subset C_{0}\left(p_{h}, h\right)$ and so $U \cap \Gamma_{3}(h) \neq \emptyset$.

Let $\mathcal{R}_{2}(f)$ and $\mathcal{R}_{3}(f)$ be the sets of continuity points of $\Gamma_{2}$ and $\Gamma_{3}$ respectively. These are residual subsets of $\mathcal{U}(f)$. We set

$$
\mathcal{R}_{U(f)}=\mathcal{R}_{0} \cap \mathcal{E}_{2} \cap \mathcal{R}_{*} \cap \mathcal{R}_{1}(f) \cap \mathcal{R}_{2}(f) \cap \mathcal{R}_{3}(f) \cap \mathcal{K} s \cap \mathcal{U}(f),
$$

which is a residual subset of $U(f)$. The next result implies our Theorems 4A and 4B.

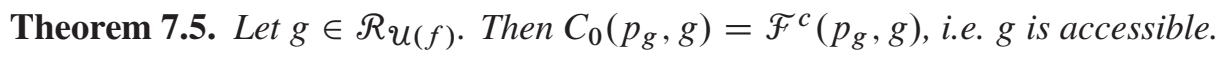

Indeed, for any $f$ as in (7.1) we consider $\mathcal{R}_{U(f)}$ as in (7.3) and we set

$$
\begin{gathered}
\mathcal{R}=\bigcup_{f \in \mathcal{R}_{0} \cap \mathcal{R} * \cap \mathcal{E}_{2} \cap \mathcal{K} s} \mathcal{R}(f) . \\
.
\end{gathered}
$$


It follows that $\mathcal{R}$ is residual (and hence $C^{r}$ dense) in $\mathcal{E}$ and every $g \in \mathcal{R}$ is accessible from Theorem 7.5. On the other hand, Corollary 5.4 implies that the accessible ones are $C^{1}$ open.

The rest of the section is thus devoted to prove Theorem 7.5.

Lemma 7.6. Let $g \in U(f)$ and let $K$ be a connected component of the boundary $\partial C_{0}\left(p_{g}, g\right)$. Then, for every $x \in K$ we have that $C_{0}(x) \subset K$.

Proof. Let $x \in K$ and let $y \in C_{0}(x)$ and assume that $y \notin K$. Since $C_{0}(x)$ is connected, we may assume, without loss of generality, that $y \notin \partial C_{0}\left(p_{g}, g\right)$. Therefore, since $y$ cannot belong to $C_{0}\left(p_{g}\right)$, we have that $y \notin \overline{C_{0}\left(p_{g}\right)}$.

On the other hand we know (by Lemma 2.7 and Remark 2.8) there is a continuous map $\gamma: B_{x} \rightarrow B_{y}$, such that for any $z \in B_{x}, \gamma(z) \in C_{0}(z)$, where $B_{x}$ and $B_{y}$ are small neighborhoods $x$ and $y$, respectively, and we may take $B_{y} \subset \mathcal{F}^{c}\left(p_{g}, g\right) \backslash$ $\overline{C_{0}\left(p_{g}\right)}$. Since $x \in \partial C_{0}\left(p_{g}\right)$ then we may take $z \in B_{x} \cap C_{0}\left(p_{g}\right)$ and hence $\gamma(z) \in C_{0}\left(p_{g}\right) \cap B_{y}$, a contradiction.

Remark 7.7. Let $g \in \mathcal{R} u(f)$ and let $K$ be a connected component of $\partial C_{0}\left(p_{g}, g\right)$. Then $K$ has no periodic point. This is because $K$ has empty interior and we know that for any periodic point $q$ of $g, C_{0}(q, g)$ is open.

Lemma 7.8. Let $g \in \mathcal{R} u(f)$ and let $h \in \mathcal{U}(f)$ such that $h=g$ in $\mathcal{F}^{c}\left(p_{g}, g\right)$. Then there is no periodic point of $h$ in $\partial C_{0}\left(p_{h}, h\right)$.

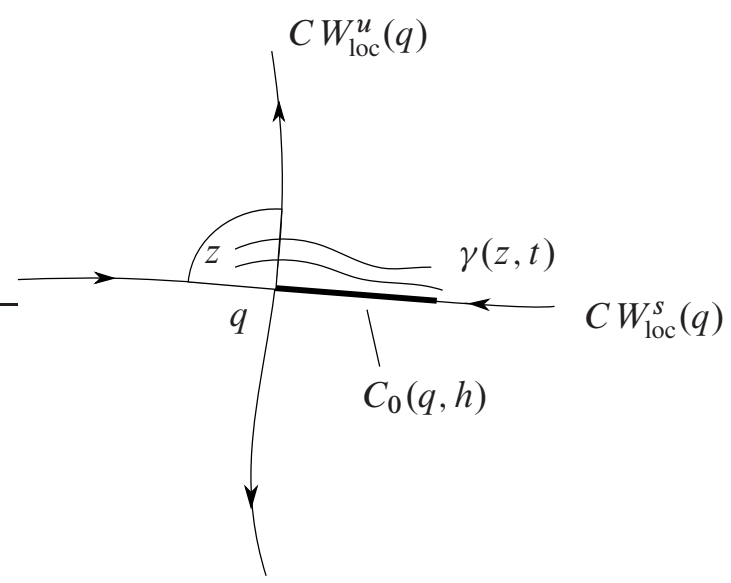

Figure 9.

Proof. Assume, by contradiction, that there exists a periodic point $q \in \partial C_{0}\left(p_{h}, h\right)$, $q \in K$ a connected component of $\partial C_{0}\left(p_{h}, h\right)$. Since $h=g$ on $\mathscr{F}^{c}\left(p_{g}, g\right)=$ $\mathscr{F}^{c}\left(p_{h}, h\right)$ we have that $q$ is a periodic point of $g$ and hence $q$ is either elliptic or 
hyperbolic (for $g_{/ \mathscr{F}^{c}}\left(p_{g}, g\right)$ and thus for $\left.h_{/ F^{c}\left(p_{g}, g\right)}\right)$. If $q$ is elliptic then we know that $C_{0}(q, h)$ is open and we get a contradiction.

Assume that $q$ is hyperbolic. If $C_{0}(q, h)$ is open we are done. If not, we know that $C W^{s}(q) \subset C_{0}(q, h)$ or $C W^{u}(q) \subset C_{0}(q, h)$. For instance, assume that $C W^{s}(q) \subset C_{0}(q, h)$. Since $h=g$ on $\mathscr{F}^{c}\left(p_{g}, g\right)$ then every periodic point of $h$ in $\mathscr{F}^{c}\left(p_{g}, g\right)$ is elliptic nondegenerated or hyperbolic and there is no saddle connections (since $g$ is $\mathcal{K} \&$ ). A theorem of J. Mather in [23, Theorem 5.2] implies that $C W^{u}(q) \subset \overline{C W^{s}(q)} \subset \overline{C_{0}(q, h)} \subset K$. We know that there exists a continuous map $\gamma: B(q) \times[0,1] \rightarrow \mathcal{F}^{c}\left(p_{g}, g\right)$ such that $\gamma(q, \cdot) \subset C W_{\mathrm{loc}}^{s}(q, h), \gamma(q, \cdot)$ is not constant and for every $z \in B(q), \gamma(z, t) \in C_{0}(z, h)$. Therefore, for $z$ belonging to an appropriate component of $B(q) \backslash\left(C W_{\mathrm{loc}}^{s}(q) \cup C W_{\mathrm{loc}}^{u}(q)\right)$ we have that $\gamma\left(z, t_{z}\right) \in$ $C W_{\text {loc }}^{u}(q)$ for some $t_{z}$ and so $z \in K$.

This implies that $K$ has nonempty interior, a contradiction.

Proposition 7.9. Let $h \in \mathcal{U}(f)$ and let $K$ be a connected component of $\partial C_{0}\left(p_{h}, h\right)$. Then, the partition of $K$ by connected component of accessibility classes form a $C^{1}$ lamination.

Proof. It is a direct consequence of Corollary 2.21.

We need a general result about $C^{1}$ lamination of subsets of the plane.

Proposition 7.10. Let $K \subset \mathbb{R}^{2}$ be a compact and connected set with empty interior and supporting a $C^{1}$ lamination. Then

(1) $\mathbb{R}^{2} \backslash K$ has at least two connected components, and

(2) if $\mathbb{R}^{2} \backslash K$ has exactly two connected component then $K \cong \mathbb{S}^{1}$.

Proof. If $K$ contains a leaf which is diffeomorphic to a circle then clearly $K$ separates $\mathbb{R}^{2}$. On the other hand, if $K$ does not contains such a leaf then by [15], $\mathbb{R}^{2} \backslash K$ has at least four connected components, this proves item 1 .

Now, if $\mathbb{R}^{2} \backslash K$ has exactly two connected components, by the above it follows that $K$ contains a leaf $W_{0}$ that is diffeomorphic to a circle, which is unique otherwise the complement has at least three connected components. Arguing by contradiction, assume that there are other leaves of the lamination than $W_{0}$.

Let $W(x)$ be the leaf of lamination through $x \in K$. Orientate the leaf in an arbitrary way. It follows that the $\alpha$ and $\omega$ limit set of the leaf must contain $W_{0}$. Otherwise, the result of [15] applies and the complement of $K$ has at least four connected components.

Consider a transversal section $J$ through $W_{0}$. By the above, every point in $K \backslash J$ is in an arc of the lamination having both ends in $J$. The same arguments in the paper of [15] yields that the lamination could be extended to a foliation with singularities in the sphere having at most one singularity of index 1 and the others have index less than $1 / 2$. It follows that the complement of $K$ has at least 3 connected components, a contradiction. 
We now state a theorem that will be useful in our context.

Theorem 7.11 (Xia [38], Koropecki [21]). Let $S$ be a compact surface without boundary and let $f: S \rightarrow S$ be a homeomorphism such that $\Omega(f)=S$. Let $K$ be a compact connected invariant set. Then, one of the following holds:

(1) K has a periodic point;

(2) $K=S=\mathbb{T}^{2}$;

(3) $K$ is an annular domain, i.e. there exists an open neighborhood $U$ of $K$ homeomorphic to an annulus and $U \backslash K$ has exactly two components (each one homeomorphic to an annulus).

Proposition 7.12. Let $g \in \mathcal{R} u(f)$ and let $h \in \mathcal{U}(f)$ such that $h=g$ on $\mathcal{F}^{c}\left(p_{g}, g\right)$. Then, any connected component $K$ of $\partial C_{0}\left(p_{h}, h\right)$ is a simple closed $C^{1}$ curve invariant for some power of $h$ (and $g$ ).

Proof. Let $K$ be a connected component of $\partial C_{0}\left(p_{h}, h\right)$. By Proposition 7.9 we know that $K$ admits a $C^{1}$ lamination. We have three possibilities:

(1) $K \subset U$ where $U$ is homeomorphic to a disk and $K$ does not separate $U$;

(2) $K \subset U$ where $U$ is homeomorphic to a disk and $K$ does separate $U$;

(3) none of the above, i.e. in any neighborhood $U$ of $K$ we have non null-homotopic closed curves (in $\mathscr{F}^{c}\left(p_{h}, h\right)$ ).

Proposition 7.10 implies that (1) cannot happen. Let's consider situation (2). We consider an open set $U_{0} \subset U$, where $U_{0}$ is a connected component of the complement of $\overline{C_{0}\left(p_{h}, h\right)}$ and $\partial U_{0} \subset K$. Since $h \mid \mathscr{F}^{c}\left(p_{h}, h\right)$ preserves the form $\omega \mid \mathscr{F}^{c}\left(p_{h}, h\right)$ we have for some integer $m$ that $h^{m}\left(U_{0}\right)=U_{0}$. This implies that $h^{m}(K) \cap K \neq \emptyset$. From the fact that $C_{0}\left(p_{h}, h\right)$ is invariant we get $h^{m}(K)=K$. Since $K$ has no periodic point (from Lemma 7.8) and is not the whole surface, we have from Theorem 7.11 that $K$ is an annular domain and by Proposition 7.10, we have that $K$ is a simple closed curve.

Finally, let's consider situation (3). Notice that there are finitely many components satisfying (3). On the other hand, $h$ maps a connected component $K$ satisfying (3), to another one also satisfying (3). Therefore, for some $m$ we have that $h^{m}(K)=K$, for any $K$ in (3). Applying Theorem 7.11 and Proposition 7.10, we get the result as before.

Lemma 7.13. Let $g \in \mathcal{R} u(f)$. Then $\overline{C_{0}\left(p_{g}, g\right)}=\mathscr{F}^{c}\left(p_{g}, g\right)$.

Proof. Assume that this is not the case, and so, there is a connected component $\mathcal{C}$ of $\partial C_{0}\left(p_{g}, g\right)$ (which is a simple closed curve) and an open annulus $U$ which is a

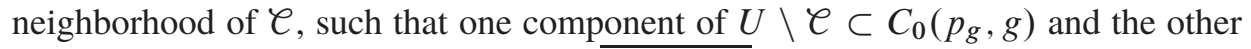
one is contained in the complement of $\overline{C_{0}\left(p_{g}, g\right)}$. We know that $g^{m}(\mathcal{C})=\ell$ for some $m$. 
Consider the family $\Gamma$ of $g^{m}$ invariant simple closed and essential $C^{1}$ curve in $U$. Notice that curves in this family are disjoint or coincide. This is because, since $g$ is $\mathcal{K} \&$, these curves cannot have rational rotation number. Now, if $\ell_{1} \cap \ell_{2} \neq \emptyset$, by invariance we have that they intersects along the nonwandering set $\Omega\left(g^{m} \mid \mathcal{C}_{1}\right)=$ $\Omega\left(g^{m} \mid \mathcal{C}_{2}\right)$. But if one (and hence both) are Denjoy maps there must exists a wandering open set $U \subset \mathscr{F}^{c}\left(p_{g}, g\right)$, a contradiction since $g$ preserves area on the compact leaf $\mathscr{F}^{c}\left(p_{g}, g\right)$.

Let $V \subset \bar{V} \subset U$ be an annulus neighborhood of $\mathcal{C}$ as in Lemma 3.7. Since $g \in \mathcal{R} u(f)$ and in particular $g$ is a continuity point of the maps $\Gamma_{2}$ and $\Gamma_{3}$ (see (7.2)) it is not difficult to see that there exists $\mathcal{V}(g)$ such that if $h \in \mathcal{V}(g)$ and $h=g$ on $\mathscr{F}^{c}\left(p_{g}, g\right)$ then $\partial C_{0}\left(p_{h}, h\right)$ must have a connected component in $V$ which must be an $h^{m}$-invariant (and so $g^{m}$-invariant) essential simple closed $C^{1}$ curve. By Lemma 3.7 we get a contradiction.

Now we are ready to finish the proof of Theorem 7.5 and hence Theorems 4A and 4B.

End of proof of Theorem 7.5. Let $g \in \mathcal{R} u(f)$ and we already know that $\overline{C_{0}\left(p_{g}, g\right)}=$ $\mathscr{F}^{c}\left(p_{g}, g\right)$. We want to prove that $C_{0}\left(p_{g}, g\right)=\mathscr{F}^{c}\left(p_{g}, g\right)$. We argue by contradiction and we assume that $C_{0}\left(p_{g}, g\right) \neq \mathscr{F}^{c}\left(p_{g}, g\right)$ and let $\boldsymbol{C}_{i}=\mathscr{C}_{i}(g)$, $i=1, \ldots, \ell$ be the connected components of $\partial C_{0}\left(p_{g}, g\right)$. We know that every $\boldsymbol{C}_{i}$ is a simple closed $C^{1}$ curve non null-homotopic invariant for $g^{m_{i}}$, for some $m_{i}$ and let $U_{i}$ be annulus neighborhoods of $\mathcal{C}_{i}$.

Since $g$ is a continuity point of $\Gamma_{2}$ and $\Gamma_{3}$ we get that there exists a neighborhood $\mathcal{V}(g)$ such that if $h \in \mathcal{V}(g)$ then

- $\overline{C_{0}\left(p_{h}, h\right)}=\mathscr{F}^{c}\left(p_{h}, h\right)$.

- $\mathscr{F}^{c}\left(p_{h}, h\right) \backslash C_{0}\left(p_{h}, h\right) \cap U_{i} \neq \emptyset, i=1, \ldots, \ell$.

Consider the family of essential simple closed $C^{1}$ curves $g^{m}$-invariant and contained in $U_{i}$ and let $V_{i}$ be as in Lemma 3.7. Since $g \in \mathcal{R} U(f)$ and for $\mathcal{V}(g)$ as above we have for any $h \in \mathcal{V}(g)$ and such that $h=g$ on $\mathscr{F}^{c}\left(p_{g}, g\right)$ that $\partial C_{0}\left(p_{h}, h\right)$ must have a connected components $C_{i}(h)$ (which are simple closed curves) contained in every $V_{i}$. By Lemma 3.7 this curves cannot be essential in $V_{i}$. This implies that $\ell_{i}(h)$ must be null-homotopic. And therefore $\overline{C_{0}\left(p_{h}, h\right)} \neq \mathscr{F}^{c}\left(p_{h}, h\right)$, a contradiction.

\section{A. Proof of Proposition 3.6}

A.1. Bounded variation. Recall that $f:[a, b] \rightarrow \mathbb{R}$ is of bounded variation if:

$$
\sup \left\{\sum_{i=0}^{n-1}\left|f\left(x_{i+1}\right)-f\left(x_{i}\right)\right|: a=x_{0}<x_{1}<\cdots<x_{n}=b\right\}<\infty,
$$

and this supremum is denoted by $V(f ;[a, b])$. 
Lemma A.1. Let $f:[a, b] \rightarrow \mathbb{R}$ of bounded variation. We have the following:

(1) if $\left[a_{1}, b_{1}\right] \subset[a, b]$ then $V\left(f ;\left[a_{1}, b_{1}\right]\right) \leq V(f ;[a, b])$,

(2) if $\left(a_{1}, b_{1}\right)$ and $\left(a_{2}, b_{2}\right)$ are disjoint intervals contained in $[a, b]$ then we have that $V\left(f ;\left[a_{1}, b_{1}\right]\right)+V\left(f ;\left[a_{2}, b_{2}\right]\right) \leq V(f ;[a, b])$. The same holds for any finite disjoint collection of intervals $\left(a_{i}, b_{i}\right)$ 's,

(3) if $\left(a_{1}, b_{1}\right)$ and $\left(a_{2}, b_{2}\right)$ are disjoint intervals in $[a, b]$ and $f\left(\left[a_{1}, b_{1}\right]\right) \cup$ $f\left(\left[a_{2}, b_{2}\right]\right) \supset[c, d]$ then $V\left(f ;\left[a_{1}, b_{1}\right]\right)+V\left(f ;\left[a_{2}, b_{2}\right]\right) \geq d-c$. A similar statement holds for any finite disjoint collection of $\left(a_{i}, b_{i}\right)$ 's,

(4) if $f$ is the difference of two non-decreasing maps then $f$ is of bounded variation.

Proof. We just prove item 3 , the others follows immediately from the definition of bounded variation. Let's assume that $c \in f\left(\left[a_{1}, b_{1}\right]\right)$. If $d \in f\left(\left[a_{1}, b_{1}\right]\right)$ then we are done. So, assume that $d \notin f\left(\left[a_{1}, b_{1}\right]\right)$ and so $d \in f\left(\left[a_{2}, b_{2}\right]\right)$. Let $c^{*}=\sup \left(f\left(\left[a_{1}, b_{1}\right]\right)\right)$ and $d^{*}=\inf \left(f\left(\left[a_{1}, b_{1}\right]\right)\right)$ then $c^{*} \geq d^{*}, c^{*} \geq c$, and $d^{*} \leq d$. Then $V\left(f ;\left[a_{1}, b_{1}\right]\right) \geq c^{*}-c$ and $V\left(f ;\left[a_{2}, b_{2}\right]\right) \geq d-d^{*}$. Then,

$$
V\left(f ;\left[a_{1}, b_{1}\right]\right)+V\left(f ;\left[a_{2}, b_{2}\right]\right) \geq\left(d-d^{*}\right)+\left(c^{*}-c\right) \geq d-c .
$$

By induction, we prove the statement for finite collections of intervals.

A.2. Proof of Proposition 3.6. Let's state it again for simplicity:

Proposition 3.6. Let $\ell_{1}:[-a, a] \rightarrow \mathbb{R}$ and $\ell_{2}:[-b, b] \rightarrow \mathbb{R}$ be two non-decreasing maps and let $\phi:[-b, b] \rightarrow[-a, a]$ be also a non-decreasing map. Then for any $\varepsilon>0$ there exist $s, t,|s|,|t| \leq \varepsilon$, such that:

$$
\phi\left(\left\{x \in[-b, b]: \ell_{2}(x)+t=x\right\}\right) \cap\left\{x \in[-a, a]: \ell_{1}(x)+s=x\right\}=\emptyset .
$$

For a non-decreasing map $f:[a, b] \rightarrow \mathbb{R}$ and $y \in[a, b]$ we denote by $f_{-}(y)=$ $\lim _{x \nearrow y} f(x)$ and $f_{+}(y)=\lim _{x \searrow y} f(x)$. We say that $f:[a, b] \rightarrow \mathbb{R}$ has a jump in $z \in(a, b)$ if $f_{-}(z) \neq f_{+}(z)$ (i.e. if $z$ is a discontinuity point of $f$ ). Moreover, we say that $f$ has a jump of size $\varepsilon$ if $f$ has a jump in some $z$ such that $\left|f_{-}(z)-f_{+}(z)\right| \geq \varepsilon$.

Lemma A.2. Let $f:[a, b] \rightarrow \mathbb{R}$ be non-decreasing. Then, for any $\varepsilon>0$ there is $\delta>0$ such that if $0<y-x<\delta$ then either $f(y)-f(x)<\varepsilon$ or there exists a jump of size $\varepsilon / 2$ between $x$ and $y$.

Proof. By contradiction, suppose that there is $\varepsilon_{0}>0$ such that for any $\delta>0$, there exist $x_{\delta}, y_{\delta}$ such that $0<y_{\delta}-x_{\delta}<\delta$ and $f\left(y_{\delta}\right)-f\left(x_{\delta}\right) \geq \varepsilon_{0}$, and there is no jump of size $\varepsilon_{0} / 2$.

Let $\left(x_{n}\right)$ and $\left(y_{n}\right)$ be two sequences such that $0<y_{n}-x_{n}<1 / n$ and $f\left(y_{n}\right)-$ $f\left(x_{n}\right) \geq \varepsilon_{0}$, and there is no jump of size $\varepsilon_{0} / 2$ between $x_{n}$ and $y_{n}$, for every $n$. Let $x$ be an accumulation point of $\left\{x_{n}\right\}$. Since $f$ is non-decreasing, we may assume that $x_{n}$ approaches $x$ from the left (otherwise $\lim f\left(y_{n}\right)-f\left(x_{n}\right)=f_{+}(x)-f_{+}(x)=0$ ). 
We may assume then that $x_{n} \nearrow x$. By the same argument, we have that $\left\{y_{n}\right\}$ has to approach $x$ from the right, and we may assume that and so $y_{n} \searrow x$. Thus, $f_{+}(x)-f_{-}(x) \geq \varepsilon_{0}$, which is a contradiction, since $x_{n} \leq x \leq y_{n}$.

Let $g_{1}:[-a, a] \rightarrow \mathbb{R}$ defined by $g_{1}=\ell_{1}(x)-x$ and $g_{2}:[-b, b] \rightarrow \mathbb{R}$ defined by $g_{2}=\ell_{2}(x)-x$. Notice that, $g_{1}, g_{2}$ are of bounded variation and that

$$
\left\{x: \ell_{1}(x)+s=x\right\}=g_{1}^{-1}(s) \quad \text { and } \quad\left\{x: \ell_{2}(x)+t=x\right\}=g_{2}^{-1}(s) .
$$

Lemma A.3. For any $s_{1}<s_{2}$ the following hold:

(1) $\overline{\phi^{-1}\left(g_{1}^{-1}\left(s_{1}\right)\right)} \cap \overline{\phi^{-1}\left(g_{1}^{-1}\left(s_{2}\right)\right)}$ contains at most finitely many points.

(2) For any $y$ in the above intersection there exists $\delta>0$ such that either

(a) $(y-\delta, y) \cap \phi^{-1}\left(g_{1}^{-1}\left(s_{1}\right)\right)=\emptyset$ and $(y, y+\delta) \cap \phi^{-1}\left(g_{1}^{-1}\left(s_{2}\right)\right)=\emptyset$, or

(b) $(y-\delta, y) \cap \phi^{-1}\left(g_{1}^{-1}\left(s_{2}\right)\right)=\emptyset$ and $(y, y+\delta) \cap \phi^{-1}\left(g_{1}^{-1}\left(s_{1}\right)\right)=\emptyset$.

Proof. Let $y \in \overline{\phi^{-1}\left(g_{1}^{-1}\left(s_{1}\right)\right)} \cap \overline{\phi^{-1}\left(g_{1}^{-1}\left(s_{2}\right)\right)}$. Observe that $\phi^{-1}\left(g_{1}^{-1}\left(s_{1}\right)\right) \cap$ $\phi^{-1}\left(g_{1}^{-1}\left(s_{2}\right)\right)=\varnothing$. We claim that $y$ cannot be accumulated at one side (either right or left) by both sets $\phi^{-1}\left(g_{1}^{-1}\left(s_{1}\right)\right)$ and $\phi^{-1}\left(g_{1}^{-1}\left(s_{2}\right)\right)$. Otherwise, assume this for the left, let $x_{n} \nearrow y, x_{n} \in \phi^{-1}\left(g_{1}^{-1}\left(s_{1}\right)\right)$ and $z_{n} \nearrow y, z_{n} \in \phi^{-1}\left(g_{1}^{-1}\left(s_{2}\right)\right)$. Then, $\phi\left(x_{n}\right) \nearrow \phi_{-}(y)$ and $\phi\left(z_{n}\right) \nearrow \phi_{-}(y)$. Hence, $s_{1}=g_{1}\left(\phi\left(x_{n}\right)\right)=\ell_{1}\left(\phi\left(x_{n}\right)\right)-\phi\left(x_{n}\right)$ and so $s_{1}=\left(\ell_{1}\right)_{-}\left(\phi_{-}(y)\right)-\phi_{-}(y)$. Analogously, $s_{2}=g_{1}\left(\phi\left(z_{n}\right)\right)=\ell_{1}\left(\phi\left(z_{n}\right)\right)-$ $\phi\left(z_{n}\right)$ and so $s_{2}=\left(\ell_{1}\right)_{-}\left(\phi_{-}(y)\right)-\phi_{-}(y)$, a contradiction since $s_{1} \neq s_{2}$. This proves item 2.

To prove item 1, let's assume that for $y$ in the intersection situation (a) holds. Then $s_{2}=\left(\ell_{1}\right)_{-}\left(\phi_{-}(y)\right)-\phi_{-}(y)$ and $s_{1}=\left(\ell_{1}\right)_{+}\left(\phi_{+}(y)\right)-\phi_{+}(y)$. So, $\left(\ell_{1}\right)_{+}\left(\phi_{+}(y)\right)=$ $s_{1}+\phi_{+}(y)$ and $\left(\ell_{1}\right)_{+}\left(\phi_{-}(y)\right)=s_{2}+\phi_{-}(y)$. Since $\left.\phi_{(} y\right) \leq \phi_{+}(y)$ and $\ell_{1}$ is nondecreasing then $\left(\ell_{1}\right)_{+}\left(\phi_{-}(y)\right) \leq\left(\ell_{1}\right)_{+}\left(\phi_{+}(y)\right)$. Then, $s_{1}+\phi_{+}(y) \geq s_{2}+\phi_{-}(y)$. Therefore,

$$
\phi_{+}(y)-\phi_{-}(y) \geq s_{2}-s_{1} .
$$

This means that the jump of $\phi$ at $y$ is at least of size $s_{2}-s_{1}$ and there are at most finitely many of them. The proof is complete in this case.

Now, assume that (b) holds and so $s_{1}=\left(\ell_{1}\right)_{-}\left(\phi_{-}(y)\right)-\phi_{-}(y)$ and $s_{2}=$ $\left(\ell_{1}\right)_{+}\left(\phi_{+}(y)\right)-\phi_{+}(y)$. Let $\varepsilon=s_{2}-s_{1}$ and let $\delta(<\varepsilon)$ from Lemma A.2 applied to $\ell_{1}$. Notices that

$$
\begin{aligned}
\left(\ell_{1}\right)_{+}\left(\phi_{+}(y)\right)-\left(\ell_{1}\right)_{-}\left(\phi_{-}(y)\right) & =s_{2}+\phi_{+}(y)-s_{1}-\phi_{-}(y) \\
& \left.=\left(s_{2}-s_{1}\right)+\phi_{+}(y)\right)-\phi_{-}(y) \geq s_{2}-s_{1} .
\end{aligned}
$$

If $y$ is a continuity point of $\phi$ then we have a $\ell_{1}$-jump of size $s_{2}-s_{1}$ at $\phi(y)=$ $\phi_{+}(y)=\phi_{-}(y)$ and there can be just finitely many of them. On the other hand, there can be finitely many $y$ 's such that the $\phi$-jump at $y$ is at least $\delta$. So, we just consider 
the set of $y$ 's such that $\phi_{+}(y)-\phi_{-}(y)<\delta$. By Lemma A.2 there exists a $\ell_{1}$-jump in $\left[\phi_{-}(y), \phi_{+}(y)\right]$ of size at least $\varepsilon / 2$. For different $y$ 's the intervals $\left[\phi_{-}(y), \phi_{+}(y)\right]$ are disjoint. Since there are finitely many $\ell_{1}$-jumps of size at least $\varepsilon / 2$, we conclude that there are finitely many $y$ 's in

$$
\overline{\phi^{-1}\left(g_{1}^{-1}\left(s_{1}\right)\right)} \cap \overline{\phi^{-1}\left(g_{1}^{-1}\left(s_{2}\right)\right)}
$$

and the lemma is proved.

Now we can prove Proposition 3.6.

Proof of Proposition 3.6. Recall that $g_{1}(x)=\ell_{1}(x)-x$ and $g_{2}(x)=\ell_{2}(x)-x$. Assume, by contradiction, that for some $\varepsilon>0$ we have that for any $s, t,|s|,|t| \leq \varepsilon$ we have

$$
\phi\left(\left\{x \in[-b, b]: \ell_{2}(x)+t=x\right\}\right) \cap\left\{x \in[-a, a]: \ell_{1}(x)+s=x\right\} \neq \emptyset .
$$

We know that $g_{2}$ is of bounded variation, set $M=V\left(g_{2} ;[-b, b]\right)$ and let $k$ be an integer, $k>M /(2 \varepsilon)$. Consider a partition of $[-\varepsilon, \varepsilon]-\varepsilon \leq s_{1}<s_{2}<\cdots<s_{k} \leq \varepsilon$, and let $S_{i}=\overline{\phi^{-1}\left(g_{1}^{-1}\left(s_{i}\right)\right)}$. Notice that from our contradicting assumption that $g_{2}\left(S_{i}\right) \supset[-\varepsilon, \varepsilon]$. From Lemma A.3 we have that $S_{i} \cap S_{j}$ contains at most finitely many points for $i \neq j$. And if $i \neq j \neq l \neq i$ then $S_{i} \cap S_{j} \cap S_{l}=\emptyset$.

Let $y_{1}, \ldots, y_{m}$ be the set of points that belongs to more than one $S_{i}$. For each $y_{i}$ let $\delta_{i}$ from Lemma A.3 such that $\left(y_{i}-\delta_{i}, y_{i}\right)$ intersects just one of the sets $S_{j}$, $j=1, \ldots, k$, and the same for $\left(y_{i}, y_{i}+\delta_{i}\right)$.

Let

$$
\widehat{S}_{j}=S_{j} \cap\left[\bigcup_{i=1}^{M}\left(y_{i}-\delta_{i}, y_{i}+\delta_{i}\right)\right]^{c} .
$$

The sets $\widehat{S}_{j}, j=1, \ldots, k$ are compact and disjoints. For each $j$, choose

$$
\widehat{U}_{j}=\bigcup_{l=1}^{m_{j}}\left[a_{l}, b_{l}\right]
$$

such that $\widehat{S}_{j} \subset \widehat{U}_{j}$ and $\widehat{U}_{j} \cap \widehat{U}_{i}=\emptyset$ if $j \neq i$.

Let

$$
U_{j}=\widehat{U}_{j} \cup\left(\bigcup_{i: S_{j} \cap\left[y_{i}-\delta, y_{i}\right] \neq \emptyset}\left[y_{i}-\delta_{i}, y_{i}\right]\right) \cup\left(\underset{i: S_{j} \cap\left[y_{i}, y_{i}+\delta\right] \neq \emptyset}{\bigcup_{i}\left[y_{i}, y_{i}+\delta_{i}\right]}\right) .
$$

We can write $U_{j}$ as a union of finitely many compact and disjoint intervals $I_{j}(1), \ldots, I_{j}\left(m_{j}\right)$. 
Now, we have:

- $g_{2}\left(U_{j}\right) \supset[-\varepsilon, \varepsilon]$ for any $j=1, \ldots, k$; and

- $\operatorname{int}\left(U_{j}\right) \cap \operatorname{int}\left(U_{l}\right)=\varnothing$ if $j \neq l$.

Therefore, we have

$$
\sum_{i=1}^{m} V\left(g_{2} ; I_{j}(i)\right) \geq 2 \varepsilon,
$$

and so, from Lemma A.1, we get

$$
V\left(g_{2} ;[-b, b]\right) \geq \sum_{j=1}^{k} \sum_{i=1}^{m} V\left(g_{2} ; I_{j}(i)\right) \geq k 2 \varepsilon>M \geq V\left(g_{2} ;[-b, b]\right),
$$

a contradiction. This completes the proof.

\section{References}

[1] A. Avila, S. Crovisier, and A. Wilkinson, Diffeomorphisms with positive metric entropy, Publ. Math. Inst. Hautes Études Sci., 124 (2016), 319-347. Zbl 1362.37017 MR 3578917

[2] A. Arbieto and C. Matheus, A pasting lemma and some applications for conservative systems. With an appendix by David Diica and Yakov Simpson-Weller, Ergodic Theory Dynam. Systems, 27 (2007), no. 5, 1399-1417. Zbl 1142.37025 MR 2358971

[3] D. V. Anosov, Geodesic flows on closed Riemannian manifolds of negative curvature, Trudy Mat. Inst. Steklov., 90 (1967), 209pp. Zbl 0176.19101 MR 224110

[4] J. Bochi, B. R. Fayad, and E. Pujals, A remark on conservative diffeomorphisms, C. R. Math. Acad. Sci. Paris, 342 (2006) no. 10, 763-766. Zbl 1097.37010 MR 2227756

[5] K. Burns, F. Rodriguez Hertz, M. A. Rodriguez Hertz, A. Talitskaya, and R. Ures, Density of accessibility for partially hyperbolic diffeomorphisms with one-dimensional center, Discrete Contin. Dyn. Syst., 22 (2008), no. 1-2, 75-88. Zbl 1154.37328 MR 2410948

[6] D. Bohnet, Codimension-1 partially hyperbolic diffeomorphisms with a uniformly compact center foliation, J. Mod. Dyn., 7 (2013), no. 4, 565-604. Zbl 1294.37013 MR 3177773

[7] K. Burns, C. Pugh, M. Shub, and A. Wilkinson, Recent results about stable ergodicity, in Smooth ergodic theory and its applications (Seattle, WA, 1999), 327-366, Proc. Sympos. Pure Math., 69, Amer. Math. Soc., Providence, RI, 2001. Zbl 1012.37019 MR 1858538

[8] K. Burns and A. Wilkinson, Stable ergodicity of skew products, Ann. Sci. École Norm. Sup. (4), 32 (1999), no. 6, 859-889. Zbl 0942.37015 MR 1717580

[9] K. Burns and A. Wilkinson, Dynamical coherence and center bunching, Discrete Contin. Dyn. Syst., 22 (2008), no. 1-2, 89-100. Zbl 1154.37332 MR 2410949

[10] K. Burns and A. Wilkinson, On the ergodicity of partially hyperbolic systems, Ann. of Math. (2), 171 (2010), no. 1, 451-489. Zbl 1196.37057 MR 2630044

[11] P. D. Carrasco, Compact dynamical foliations, Ergodic Theory Dynam. Systems, 35 (2015), no. 8, 2474-2498. Zbl 1356.37046 MR 3456603 
[12] S. Crovisier, Dynamics of $C^{1}$-diffeomorphisms: global description and prospects for classification, in Proceedings of the International Congress of Mathematicians (Seoul, Korea, 2014), III, 571-595, Kyung Moon SA Co. Ltd., 2014. Zbl 06433088

[13] B. Dacorogna and J. Moser, On a partial differential equation involving the Jacobian determinant, Ann. Inst. H. Poincaré Anal. Non Linéaire, 7 (1990), no. 1, 1-26. Zbl 0707.35041 MR 1046081

[14] D. Dolgopyat and A. Wilkinson, Stable accessibility is $C^{1}$ dense. Geometric methods in dynamics. II, Astérisque, (2003), no. 287, xvii, 33-60. Zbl 1213.37053 MR 2039999

[15] R. J. Fokkink and L. G. Oversteegen, The geometry of laminations, Fund. Math., 151 (1996), no. 3, 195-207. Zbl 0880.54024 MR 1424574

[16] J. Franks, Recurrence and fixed points of surface homeomorphisms, Ergodic Theory Dynam. Systems, 8*, Charles Conley Memorial Issue (1988), 99-107. Zbl 0634.58023 MR 967632

[17] A. Gogolev, Partially hyperbolic diffeomorphisms with compact center foliations, J. Mod. Dyn., 5 (2011), no. 4, 747-769. Zbl 1254.37029 MR 2903756

[18] M. Grayson, C. Pugh, and M. Shub, Stably ergodic diffeomorphisms, Ann. of Math. (2), 140 (1994), no. 2, 295-329. Zbl 0824.58032 MR 1298715

[19] E. Hopf, Statistik der geodätischen Linien in Mannigfaltigkeiten negativer Krümmung, Ber. Verh. Sächs. Akad. Wiss. Leipzig, 91 (1939), 261-304. Zbl 0024.08003 MR 1464

[20] M. Hirsch, C. Pugh, and M. Shub, Invariant manifolds, Lect. Notes in Math., 583, Springer Verlag, 1977. Zbl 0355.58009 MR 501173

[21] A. Koropecki, Aperiodic invariant continua for surface homeomorphisms, Math. Z., 266 (2010), no. 1, 229-236. Zbl 1218.37048 MR 2670681

[22] J. Lewowicz, Lyapunov functions and topological stability, J. Differential Equations, 38 (1980), no. 2, 192-209. Zbl 0418.58012 MR 597800

[23] J. Mather, Invariant subsets for area preserving homeomorphisms of surfaces, in Mathematical analysis and applications, Part B, 531-562, Adv. in Math. Suppl. Stud., 7, Academic Press, New York, 1981. Zbl 0505.58027 MR 634258

[24] J. Moser, On the volume elements on a manifold, Trans. Amer. Math. Soc., 120 (1965), 286-294. Zbl 0141.19407 MR 182927

[25] C. Pugh and M. Shub, Stable ergodicity and partial hyperbolicity, in International Conference on Dynamical Systems (Montevideo, 1995), 182-187, Pitman Res. Notes Math. Ser., 362, Longman, Harlow, 1996. Zbl 0867.58049 MR 1460804

[26] C. Pugh and M. Shub, Stably ergodic dynamical systems and partial hyperbolicity, J. Complexity, 13 (1997), no. 1, 125-179. Zbl 0883.58025 MR 1449765

[27] C. Pugh and M. Shub, Stable ergodicity and julienne quasi-conformality, J. Eur. Math. Soc. (JEMS), 2 (2000), no. 1, 1-52. Zbl 0964.37017 MR 1750453

[28] C. Pugh, M. Shub, and A. Wilkinson, Hölder foliations, Duke Math. J., 86 (1997), 517-546. Zbl 0877.58045 MR 1432307

[29] F. Rodriguez Hertz, Stable ergodicity of certain linear automorphisms of the torus, Ann. of Math. (2), 162 (2005), no. 1, 65-107. Zbl 1098.37028 MR 2201693 
[30] F. Rodriguez Hertz, M. A. Rodriguez Hertz, A. Tahzibi, and R. Ures, A criterion for ergodicity of non-uniformly hyperbolic diffeomorphisms, Electron. Res. Announc. Math. Sci., 14 (2007), 74-81. Zbl 1139.37015 MR 2353803

[31] F. Rodriguez Hertz, M. A. Rodriguez Hertz, and R. Ures, A survey of partially hyperbolic dynamics, in Partially hyperbolic dynamics, laminations, and Teichmüller flow, 35-87, Fields Inst. Commun., 51, Amer. Math. Soc., Providence, RI, 2007. Zbl 1149.37021 MR 2388690

[32] F. Rodriguez Hertz, M. A. Rodriguez Hertz, and R. Ures, Accessibility and stable ergodicity for partially hyperbolic diffeomorphisms with 1D-center bundle, Invent. Math., 172 (2008), no. 2, 353-381. Zbl 1136.37020 MR 2390288

[33] D. Repovš, A. B. Skopenkov, and E. V. Ščepin, $C^{1}$-homogeneous compacta in $\mathbf{R}^{n}$ are $C^{1}$-submanifolds of $\mathbf{R}^{n}$, Proc. Amer. Math. Soc., 124 (1996), no. 4, 1219-1226. Zbl 0863.53004 MR 1301046

[34] M. Shub, Topologically transitive diffeomorphisms on $T^{4}$, Springer Lecture Notes in Mathematics, 206, 39-40, Springer Verlag, 1971.

[35] M. Shub and A. Wilkinson, Stably ergodic approximation: two examples, Ergodic Theory Dynam. Systems, 20 (2000), no. 3, 875-893. Zbl 0970.37022 MR 1764933

[36] A. Weinstein, Symplectic manifolds and their Lagrangian submanifolds, Advances in Math., 6 (1971), 329-346. Zbl 0213.48203 MR 286137

[37] A. Wilkinson, Conservative partially hyperbolic dynamics, in Proceedings of the International Congress of Mathematicians. Volume III, 1816-1836, Hindustan Book Agency, New Delhi, 2010. Zbl 1246.37054 MR 2827868

[38] Z. Xia, Area-preserving surface diffeomorphisms, Comm. Math. Phys., 263 (2006), no. 3, 723-735. Zbl 1103.37029 MR 2211821

[39] Z. Xia and H. Zhang, A $C^{r}$ closing lemma for a class of symplectic diffeomorphisms, Nonlinearity, 19 (2006), no. 2, 511-516. Zbl 1134.37351 MR 2199401

[40] E. Zehnder, Homoclinic points near elliptic fixed points, Comm. Pure Appl. Math., 26 (1973), 131-182. Zbl 0261.58002 MR 345134

[41] Z. Zhang, $C^{r}$ density of stable ergodicity for a class of partially hyperbolic systems, 2015. arXiv: 1507.03556

Received March 07, 2016; revised October 14, 2016

V. Horita, Departamento de Matemática, IBILCE/UNESP,

Rua Cristóvão Colombo 2265, 15054-000 S. J. Rio Preto, SP, Brazil

E-mail: vhorita@ibilce.unesp.br

M. Sambarino, CMAT, Facultad de Ciencias, Universidad de la República, Iguá 4225, Montevideo, Uruguay

E-mail: samba@cmat.edu.uy 